Boise State University

ScholarWorks

$6-2020$

\title{
Quantifying the Relative Importance of Variation in Predation and the Environment for Species Coexistence
}

Lauren G. Shoemaker

University of Wyoming

Allison K. Barner

University of California, Berkeley

Leonora S. Bittleston

Boise State University

Ashley I. Teufel

Santa Fe Institute 


\section{Quantifying the relative importance of variation in predation and the environment for species coexistence}

Lauren G. Shoemaker ${ }^{1}$, corresponding author email: lshoema1@uwyo.edu, phone: (970)-691-0459, fax: NA

${ }^{2}$ Botany Department, University of Wyoming, Laramie, WY, 82071

Allison K. Barner ${ }^{2,3}$, corresponding author email: allison.barner@colby.edu, fax: NA

${ }^{2}$ Department of Environmental Science, Policy, and Management

University of California, Berkeley, Berkeley, CA, 94720

${ }^{3}$ Department of Biology, Colby College, Waterville, ME, 04901

Leonora S. Bittleston ${ }^{4,5}$

email: leonorabittleston@boisestate.edu

${ }^{4}$ Department of Civil and Environmental Engineering, Massachusetts Institute of Technology, Cambridge, MA, 02139

${ }^{5}$ Department of Biological Sciences, Boise State University, Boise, ID, 83725

Ashley I. Teufel ${ }^{6,7}$

email: ateufel@santafe.edu

${ }^{6}$ Santa Fe Institute, Santa Fe, NM, 87501

${ }^{7}$ Department of Integrative Biology, The University of Texas at Austin, Austin, TX, 78712

Data accessibility statement: All code to replicate this study can currently be found on GitHub at https://github.com/lash1937/foodwebs_env_variation_coexistence. Upon acceptance, code will be archived on Zenodo.

Running Title: Environment and predation affect coexistence

Keywords: Coexistence theory, ecological networks, species interactions stabilizing mechanisms, environmental fluctuations, diamond model, storage effect

Type of Article: Letters 
This is an author-produced, peer-reviewed version of this article. The final, definitive version of this document can be found online at Ecology Letters, published by John Wiley \& Sons Ltd/CNRS. Copyright restrictions may apply. doi: 10.1111/ele.13482

Number of Words: Abstract: 150, Main text: 4787, Text Box: 683

Number of References: 66

Number of Tables: 1

Number of Figures: 4

Number of Text Boxes: 1

Statement of authorship: LGS and AKB contributed equally to this manuscript and led the working group from which this project originated. All authors contributed to conceptual development, analyses, manuscript writing, and editing. 


\section{Abstract}

Coexistence and food web theory are two cornerstones of the longstanding effort to understand how species coexist. Although competition and predation are known to act simultaneously in communities, theory and empirical study of these processes continue to be developed largely independently. Here, we integrate modern coexistence theory and food web 6 theory to simultaneously quantify the relative importance of predation and environmental 7 fluctuations for species coexistence. We first examine coexistence in a theoretical multi8 trophic model, adding complexity to the food web using machine learning approaches. We 9 then apply our framework to a stochastic-difference equation model of the rocky intertidal

food web, partitioning empirical coexistence dynamics. We find the main effects of both environmental fluctuations and variation in predator abundances contribute substantially to species coexistence. Unexpectedly, their interaction tends to destabilize coexistence, leading to new insights about the role of bottom-up versus top-down forces in both theory and the rocky intertidal ecosystem. 
 \\ 2 Introduction}

For many decades, community ecologists have developed two complementary theoretical and empirical directions for studying the interactions among species and their dynamic consequences: food web theory (Cohen \& Stephens, 1978; McCann, 2011; Terborgh, 2015) and coexistence theory (Chesson, 2000; Barabás et al., 2018). Despite their long independent histories, central to both approaches is a shared interest in explaining the mechanisms that maintain biological diversity, coexistence, and the stability of ecological communities (Ives et al., 2005; Godoy et al., 2018).

Food web theory focuses on consumptive interactions (links) between species across different trophic levels (illustrated as arrows pointing in the direction of energy flow in Fig. 1A). In this framework, competition between species results from competitors sharing limiting resources (e.g., nutrients, energy, space Godoy et al. (2018)). Empirical studies of food webs tend to quantify the presence or absence of links between species at different trophic levels or the frequency of consumptive events among all species in the food web (Berlow et al., 2004; Pascual et al., 2006). From theoretical studies of food webs, we have gained the insight that these "top-down" forces can promote coexistence of species at lower trophic levels and that diverse ecological communities are stabilized by weak interactions between species (McCann et al., 1998) as well as negative feedbacks (May, 1973).

In comparison, coexistence theory tends to focus on competitive interactions within a single trophic level, exploring how multiple species competing for the same limiting resources, space, or responding to environmental fluctuations, can coexist (illustrated as a wide, double-pointed arrow in Fig. 1B). Classic coexistence work shows that diverse and stable communities can occur through three primary mechanisms: (1) the partitioning of resources, where different species are better able to take advantage of different limiting resources, such as nitrogen versus phosphorous in grasslands (Tilman, 1982) (2) trait and 
demographic trade-offs between species, such as if one species has a higher dispersal rate while another is a superior competitor (Levins \& Culver, 1971; Yu \& Wilson, 2001), and (3) species partitioning environmental heterogeneity and inherent landscape-level variation, stabilizing overall community dynamics (Chesson, 2000). Current developments in coexistence theory tend to highlight how variation in these environmental "bottom-up" processes and niche partitioning can promote species coexistence via different mechanisms, the most widely studied of which is the storage effect (Chesson, 2000; Snyder \& Adler, 2011; Barabás et al., 2018). Empirical applications of this coexistence framework - termed modern coexistence theory (MCT) - tend to quantify demographic rates under different environmental conditions to examine the relative importance of multiple coexistence mechanisms (Kraft et al., 2015; Germain et al., 2018).

Predation and competition are key forces structuring communities, and at their cores, clear connections exist between these two robust theories for community ecology. Both examine nearly identical questions such as: (1) Why do we observe diverse communities, rather than having one or only a few species dominate? and (2) What mechanisms promote coexistence of species with one another? Past work integrating food webs and coexistence generally fall into three categories: (1) the influence of predators (or "natural enemies") on the diversity of prey species (Jabot \& Bascompte, 2012; Saleem et al., 2012; Griffin et al., 2013), (2) how predator presence alters the strength of competition among prey species (Gurevitch et al., 2000), and (3) coexistence of prey species, as mediated by predation (Holt, 1984; Holt et al., 1994; Chesson \& Kuang, 2008; McPeek, 2019; Klauschies \& Gaedke, 2019). Despite the breadth of previous work, few studies have incorporated known food web structure with realistic complexity. The best known example, Brose (2008) implements a consumer-resource model for a diverse simulated food web and analyzes the conditions for persistence in consumers and resources, using an analog of Tilman's $\mathrm{R}^{*}$ theory of limiting resources (Tilman, 1982). While predation has been explicitly incorporated into modern coexistence theory in 
select scenarios (Chesson \& Kuang, 2008; Kuang \& Chesson, 2008, 2009, 2010), the approach relies on analytical derivations that are only possible under simplifying assumptions and relatively small food webs, making it difficult to generally apply the framework across more complex theoretical examples, much less empirical systems (Ellner et al., 2019).

Here, we seek to explicitly combine food web theory and coexistence theory, integrating the joint role of predation and environmental variability for coexistence in a conceptual and mathematical framework that is generalizable, easy to use, and can be applied across different theoretical and empirical systems. Furthermore, the framework allows for a direct quantification of the relative importance of each mechanism to overall stable coexistence, or alternatively to competitive exclusion of specific species. To do so, we extend a recent conceptualization of modern coexistence theory (MCT) (Ellner et al., 2019) to examine how fluctuations in the environment (bottom-up processes), fluctuations in predator abundance (top-down processes), their interaction, and average fitness differences between competitors can stabilize - or alternatively hinder - coexistence.

\section{Methods}

We extended MCT to quantify under what conditions species coexist and the mechanisms that promote stable coexistence. Extending MCT, we decomposed coexistence into four mechanisms (Box 1) that quantify (1) the role of fluctuation independent mechanisms, $\Delta_{i}^{0}$, (2) the role of bottom-up fluctuations, $\Delta_{i}^{E}$, the role of top-down fluctuations, $\Delta_{i}^{P}$, and their interaction, $\Delta_{i}^{E P}$. This decomposition can be applied across food webs. Here, we first decomposed coexistence into its mechanistic components, in a simple but highly studied diamond-shaped food web (McCann et al., 1998; Vasseur \& Fox, 2007). Doing so allowed us to compare the relative importance of fluctuations in the environment versus predators for the coexistence of two competing species. We then examined the generality of coexistence partitioning across parameter space, extending this framework to incorporate additional 
This is an author-produced, peer-reviewed version of this article. The final, definitive version of this document can be found online at Ecology Letters, published by John Wiley \& Sons Ltd/CNRS. Copyright restrictions may apply. doi: 10.1111/ele.13482

91 food web complexity via added competitors and predators. Finally, we applied our approach

to a classic empirical ecosystem - the rocky intertidal food web-highlighting its utility in empirical scenarios and insights gained in both theoretical and empirical cases.

\subsection{Diamond model}

Applying the framework from Box 1, we first examined the relative importance of environmental fluctuations versus fluctuations in predator abundance using a four-species diamond model (Fig. 1A). The diamond model tracks abundance of a top predator, $P$, two competitors, $C_{1}$ and $C_{2}$, and a resource, $R$. Competitor 1 is the superior competitor, but is also the preferred prey species, which maintains coexistence under a variety of parameterizations. This classic model has a long history for analyses of trophic interactions and species coexistence, including in identifying the stabilizing effect of competitor asynchrony in constant environments (McCann et al., 1998) and with extensions explicitly incorporating environmental fluctuations (Vasseur \& Fox, 2007). Furthermore, in the model, competitors share resources and predators, matching common empirical systems (Williams \& Martinez, 2000) in a mathematically simplified and tractable manner. In the model, dynamics occur such that:

$$
\begin{aligned}
& \frac{d P}{d t}=-M_{P} P+\frac{J_{P} P\left[\Omega_{P C_{1}} C_{1}+\left(1-\Omega_{P C_{1}}\right) C_{2}\right]}{\Omega_{P C_{1}} C_{1}+\left(1-\Omega_{P C_{1}}\right) C_{2}+C_{0}} \\
& \frac{d C_{1}}{d t}=-M_{C_{1, t}} C_{1}+\frac{\Omega_{C_{1} R} J_{C_{1}} C_{1} R}{R+R_{0_{1}}}-\frac{\Omega_{P C_{1}} J_{P} P C_{1}}{\Omega_{P C_{1}} C_{1}+\left(1-\Omega_{P C_{1}}\right) C_{2}+C_{0}} \\
& \frac{d C_{2}}{d t}=-M_{C_{2, t}} C_{2}+\frac{\Omega_{C_{2} R} J_{C_{2}} C_{2} R}{R+R_{0_{2}}}-\frac{\left(1-\Omega_{P C_{1}}\right) J_{P} P C_{2}}{\Omega_{P C_{1}} C_{1}+\left(1-\Omega_{P C_{1}}\right) C_{2}+C_{0}} \\
& \frac{d R}{d t}=r R(1-R / K)-\frac{\Omega_{C_{1 R}} J_{C_{1}} C_{1} R}{R+R_{0_{1}}}-\frac{\Omega_{C_{2 R}} R_{C_{2}} J_{C_{2}} C_{2} R}{R+R_{0_{2}}} .
\end{aligned}
$$


This is an author-produced, peer-reviewed version of this article. The final, definitive version of this document can be found online at Ecology Letters, published by John Wiley \& Sons Ltd/CNRS. Copyright restrictions may apply. doi: 10.1111/ele.13482

where parameter definitions and values are given in Table 1. Disparities in consumption of the resource and predator preference yields asymptotic dynamics where competitor populations are highly asynchronous and both species co-occur (Vasseur \& Fox, 2007).

Environmental variation alters competitor mortality rates, $M_{C_{s, t}}=M_{C_{s, 0}} \cdot e^{\zeta_{s, t}}$, where $\zeta_{s, t}$ are random normally distributed environmental conditions. For the two competitor species, $s$, a time series of environmental effects $\zeta$ is calculated using the Cholesky factorization of the variance-covariance matrix:

$$
\left[\begin{array}{cc}
\sigma^{2} & \rho \sigma^{2} \\
\rho \sigma^{2} & \sigma^{2}
\end{array}\right] .
$$

where $\sigma$ is the environmental effect size and $\rho$ is the cross-correlation of its effect on competitor species. Multiplying Eq. 5 by a 2 X $T$ matrix of random numbers from a normal distribution with mean 0 and unit variance, where $T$ is the total number of timesteps to run the model $(T=5000)$, yields $\zeta_{1, t}$ and $\zeta_{2, t}$ for the two competitor species and each timestep $t$.

Applying MCT partitioning to the diamond model, we calculated growth rate when rare and its mechanistic decomposition for each competitor species. As an intermediate step, we examined results with no environmental fluctuations, setting $\sigma=0.0$. We then examined the combined effect of fluctuations in the environment and predator abundances, setting $\sigma=0.55$ and comparing scenarios with negative $(\rho=-0.75)$, no $(\rho=0)$, and positive ( $\rho=0.75)$ cross-correlation, as environmental correlation in variability can exhibit large ramifications for community properties (Ruokolainen et al., 2009; Shoemaker et al., 2019). Finally, we determined the relative importance of top-down versus bottom-up controls by simultaneously varying the predation preference (the top-down control) and the strength of environmental fluctuations (the bottom-up control). Specifically, we varied predator preference from $\Omega_{P C_{1}}=0.5$ (no preference) to $\Omega_{P C_{1}}=1.0$ (full preference) along with varying 
the strength of environmental fluctuations from $\sigma=0$ to $\sigma=0.75$. This spans the range of observed environmental fluctuation effects on mortality in both terrestrial and aquatic systems (Vasseur \& Fox, 2007; Condit et al., 1995). For each parameter combination, we calculated each species' growth rate when rare, determining coexistence using the mutual invasibility criterion. Then, using an example with strong predation preference $\left(\Omega_{P C_{1}}=0.9\right)$, we decomposed coexistence into its mechanistic components for low, intermediate, and high environmental variability ( $\sigma=0.1,0.4$ and 0.7 respectively, Supplement 2). All simulations and analyses were conducted in R (R Core Team, 2019).

\subsection{Expanded diamond model}

While the diamond model represents a natural starting-point for examining coexistence, it is a highly simplified food web structure. We therefore expanded the model, first by incorporating a third competitor $\left(C_{3}\right)$ (equation details in Supplement 3, Eq. S3.1). In including a third competitor, we maintained the initial diamond model parameterization except for predator preferences, and applied global optimization by differential evolution to assign values to the new parameters $M_{C_{3}}, J_{C_{3}}, R_{0_{3}}, \Omega_{P C_{1}}, \Omega_{P C_{2}}$, and $\Omega_{C_{2} R}$ and predator preferences (Table 1). To maintain similar dynamics of the predator from the initial diamond model we bounded estimates of the predators prey preference such that these values could only differ by up to $1 / 3$ of the values from the initial diamond model. To ensure that populations were stable, we assigned parameters without stochastic mortality of competitors. We created a scoring function based on counting the number of instances any of the populations fell below a value of 0.001 over the course of 5000 time steps (excluding the first 1000 'burn-in' time points). A value of zero in the scoring function reflects population dynamics where each of the species coexist over the course of the time series. We applied differential evolution to minimize the scoring function using the $\mathrm{R}$ package DEoptim (Ardia et al., 2016), with the $\mathrm{DE} /$ best / 1 / bin with jitter (option 3), an initial population size of 100 individuals, and a 0.05 speed of the crossover adaptation. We ran the algorithm until the scoring function 
reached zero and recorded the parameter set from the first member of the population of solutions given from the algorithm. Since differential evolution is a stochastic algorithm, we ran the algorithm again to generate a second set of parameters that also result in the coexistence of each of the species. Repeating this process twice allowed us to examine if coexistence mechanisms differ under an alternative parameter combination with the same food web topology (Table 1). We visually confirmed that both of the parameter sets resulted in different dynamics in the absence of environmental variation (Fig. S3.1, S3.2) and with environmental variation (Fig. S3.3, S3.4). Using these systems, we calculated coexistence and its mechanistic decomposition when including environmental variation as described above.

To further investigate how complex systems are stabilized, we included a second predator in the model (Eq. S3.2). We followed the same method as above, using the parameter set of replicate 1 from Table 1 to assign the new parameters for the second predator. We bounded the second predators preference coefficient such that they must be larger than 0.05 for each competitor to ensure energy flow across both predation pathways. We visually confirmed that the estimated parameter set for the model containing two predators and three competitors resulted in stable dynamics (Fig. S3.5, Fig. S3.6). This methodology allowed us to compare coexistence mechanisms with the entire food web (two predators, three competitors, and one resource) and a subset of species from the food web.

\subsection{Empirical applications in an intertidal food web}

Finally, we highlight the applicability of MCT partitioning (Box 1) in empirical systems, focusing on the rocky intertidal ecosystem (Fig. 1D), although the framework is applicable across systems. The rocky intertidal communities of the Northeast Pacific Ocean are wellstudied model systems for exploring the role of predation and environmental variation in species coexistence (Connell, 1961; Dayton, 1971; Menge et al., 1997; Connolly \& Roughgarden, 1999; Forde \& Doak, 2004). In this system, a larger barnacle Balanus glandula competes for space with the smaller barnacle Chthamalus dalli/fissus and with herbivorous limpets 
(Dayton, 1971). Whelks (predatory snails) and sea stars consume both barnacles, but are not space-limited like barnacles and limpets. Of these five focal taxa, four have planktonic larvae (sea stars, barnacles, limpets), which leads to a decoupling of local population abundance and recruitment (Iwasa \& Roughgarden, 1986). Coexistence among sessile, space occupying invertebrates in this system is thought to be controlled both by 'keystone' predation (Paine, 1966, 1969) and bottom-up variation in larval supply (Menge et al., 1997). These processes operate at different spatial scales, with predation occurring at the local scale, while environmental variation altering recruitment originates at the regional scale through variation in oceanographic patterns such as upwelling intensity. It remains unclear the degree to which each mechanism - and thus each spatial scale - contributes to coexistence.

Model. We modeled the rocky intertidal food web (Fig. 1D) using a set of stochastic difference equations, based closely on the model and parameterization of Forde \& Doak (2004). While here we summarize the model, the full set of equations (Eq. S4.1-S4.10) and parameterization (Table S4.1) can be found in Supplement 4. In brief, the model tracks recruitment dynamics, competition for space, and predator-prey interactions through time. First, the pelagic larval pool for each species (barnacles, limpets, and sea stars) is randomly drawn from a lognormal distribution, based on the range of observed values for the system, mimicking the spatial and temporal recruitment variation in coastal ecosystems (Menge et al., 1997). Next, the realized recruitment from this larval pool to the local ecosystem depends on the availability of free space. Following Shinnen \& Navarrete (2014) and Forde \& Doak (2004), since no clear competitive hierarchy exists for the rocky intertidal (Menge, 2000), we model competition for free space using lottery competition, where the available free space in the system is calculated at each time step (month) based on the individual size and population abundances of the space-occupiers.

Both recruits and adults of all species are affected by density-independent mortality, while barnacles face additional mortality dependent on predator population size. Predators have 
the same per-capita effect on both barnacles. In this model, neither prey is preferred over the other (Connolly \& Roughgarden, 1999; Forde \& Doak, 2004), though predator preference for Balanus glandula has been observed (Dayton, 1971) and could be modeled in a future study. Population growth of limpets, sea stars, and whelks is density-dependent, as their predators are not explicitly modeled (Supplement 4).

We simulated the rocky intertidal model 500 times, each for 100 years, tracking population size for each species per month (Forde \& Doak, 2004). We examined coexistence across six larval supply scenarios, paralleling previous analyses (e.g. Forde \& Doak (2004)) and encompassing observed different laraval supply rates due to spatiotemporal differences in upwelling intensity and other oceanographic factors (Menge et al., 1997; Menge, 2000). First, we compared coexistence when all species with pelagic larvae had 'high' versus 'low' mean supply rates, then subsequently each species was run individually with 'high' supply, while all others were held at 'low' supply rates (see Table 1 in Forde \& Doak, 2004). Coexistence mechanisms were calculated following the procedure described in Box 1 and Supplement 1. Invasion population size was set to 1 for all species, and invasion was simulated after 50 years, to ensure that resident species reached their steady state distribution. Here, variation in the environment manifests as variation in larval supply rather than mortality (as in the diamond model) and relative nonlinearity in predation, $\Delta_{i}^{P}$, includes variation in predator abundance and recruitment of predators.

\section{Results}

\subsection{Diamond model}

When decomposing coexistence into its mechanistic components for the classic diamond model (Fig. 2A), we find that both competitors are able to stably coexist, as both exhibit positive growth rates when rare, $\bar{r}_{i}-\bar{r}_{r}$ (Fig. 2B,C). For the superior competitor (Fig. 2B), fluctuations in either the environment or the predator abundance matters little for 
coexistence, as evidenced by the similarity in strength of $\bar{r}_{i}-\bar{r}_{r}$ and $\Delta_{i}^{0}$, as well as the minimal effects of $\Delta_{i}^{E}, \Delta_{i}^{P}$, and $\Delta_{i}^{E P}$. This result is consistent across scenarios with and without environmental variation (Fig. 2B,C and S2.1) However, for the inferior competitor (Fig. $2 \mathrm{C})$, fluctuations in predator abundance stabilize coexistence when environmental variation is absent (Fig. S2.1), and fluctuations in both predator abundance and the environment stabilize coexistence when $\sigma=0.55$. Fluctuation independent mechanisms are positive for both species due to tradeoffs between resource use and predator preference. Additionally, high predator abundances preferentially increase consumption and decrease the steady stateabundance of the superior competitor, $C_{1}$. This decrease in competition between $C_{1}$ and $C_{2}$ increases the stability of coexistence for the inferior competitor $\left(C_{2}\right)$ via relative nonlinearity in predation. Similarly, relative nonlinearity in response to the environment increases the growth rate when rare for the inferior competitor. However, the interactive effect between environmental fluctuations and fluctuations in predator abundance is destabilizing for $C_{2}$. This destabilization of $\Delta_{i}^{E P}$ occurs because random fluctuations in the environment and mortality of $C_{1}$ lead to an increase in the average predator abundance (approximately 20\%), yielding a negative interaction effect of environment and predator fluctuations on $C_{2}$. These results are robust to changes in the cross-correlation of environmental fluctuations (Figs. S2.2 and S2.3).

To examine the generality of these results, we calculated coexistence when varying predation preference and the strength of environmental variation simultaneously. Predation preference had a stronger effect on both coexistence and growth rates when rare compared to the strength of environmental fluctuations (Fig. 3). With no predation preference, $C_{1}$ outcompetes the inferior competitor $C_{2}$. As predation preference increases, $C_{2}$ is then able to outcompete $C_{1}$. Only at high preference of the predator for $C_{1}$ do both species coexist, as the high predation preference yields oscillatory dynamics that maintain coexistence.

However, while growth rates when rare for each species and overall coexistence depend 
only moderately on the strength of environmental variation, the relative importance of coexistence mechanisms changes substantially with increased environmental variation (Fig. S2.4). With low environmental variation, coexistence of $C_{2}$ with $C_{1}$ is stabilized by $\Delta_{i}^{0}$ and $\Delta_{i}^{P}$. As the strength of environmental variation increases, $\Delta_{i}^{0}$ becomes less important and even destabilizing at high environmental variation. Similarly, $\Delta_{i}^{E P}$ becomes increasingly destabilizing, while $\Delta_{i}^{P}$ and $\Delta_{i}^{E}$ become more stabilizing. The combination of these mechanisms yields coexistence regardless of the amount of environmental variation, $\sigma$, but highlights that the relative importance of coexistence mechanisms changes with increasing environmental variation and that their effects often counteract one another.

\subsection{Expanded diamond model}

To assess how coexistence changes with increasing food web complexity, we first examined adding another competitor to the diamond model (Fig. 2D-G). We find that the inclusion of a third competitor causes an increase in the stabilization due to predation and environmental variation for the superior competitor (Fig. 2E). Inclusion of the third competitor also results in a destabilization by variation in predation in the second competitor (Fig. 2F). Further, comparing the same model, but with two parameter sets, highlights differing stabilizing mechanisms for $C_{1}$ (Fig. S3.7E,I), where the effect of predator and environmental variation can be stabilizing (Fig. S3.7E) or destabilizing (Fig. S3.7I). These findings suggest that the stability of a food web is achieved not just through its structure, but as a function of how the species interact with one another. Furthermore, as the classic diamond model represents a subset of this larger food web (with the same parameterization), comparing Fig. 2B-C, E-G and Fig. S3.7 highlights the different expectations for coexistence when only considering part of the larger ecological community.

To compare how mechanisms of stabilization change when a second predator is included (Fig. 2H) we decompose the coexistence mechanisms of this expanded system (Fig. 2I-K). The second predator increases stabilization of growth rate when rare by relative nonlinearity in 
predation for $C_{2}$ and causes the effect of $\Delta_{i}^{E P}$ to switch from stabilizing to destabilizing (Fig. $2 \mathrm{~F}, \mathrm{~J})$. We also find that the second predator increases the the amount of stabilization of $C_{3}$ due to predation (Fig. 2G,K) Again, we find different expectations for coexistence strength and its mechanistic comparison when comparing the full model to ones that only consider a subset of species interactions. In aggregate, our results from decomposing increasingly complex food webs demonstrate that the nature of interactions as well the food web topology impact the mechanism by which species coexist.

\subsection{Empirical applications in an intertidal food web}

Finally, we examined coexistence in a temperate rocky intertidal ecosystem, a classically studied system in which both predation and environmental variation have been shown to influence species coexistence. Here, variation in the environment (e.g. regional processes, such as upwelling intensity) drives variation in larval supply rates of the three taxa that compete for space. With low environmental variation, the top competitor is the smaller barnacle, Chthamalus dalli, though both barnacle species coexist (Fig. 4 top row). When environmental variation is high, across all planktonic taxa (barnacles, limpets, sea stars), this variation in larval supply benefits both barnacle taxa, as evidenced by the increase in both species' growth rates when rare (Fig. 4 bottom row). Relative nonlinearity in response to the environment is the strongest mechanism promoting coexistence while relative nonlinearity in predation also promotes coexistence of barnacle prey, though to a lesser extent than variation in the environment. Higher positive invasion growth rates under predation suggests a potential 'hydra effect' of sea stars and whelks on their barnacle prey (Abrams, 2009). However, coexistence is strongly destabilized by covariation in predator abundance and environmental conditions, especially with high environmental variability, paralleling Fig. 2B,C.

When larval supply is low, covariation in the environment (larval supply) and predation $\left(\Delta_{i}^{E P}\right)$ greatly benefits limpets, who have no predators in this model. In other words, limpets benefit from the co-occurrence of high predator abundance and high larval supply, as high 
311 predator abundance reduces the abundance of species that compete with limpets, though

not enough to allow limpets to ultimately coexist (Fig. 4C,F). In fact, across all scenarios, limpets do not have a positive growth rate when rare (Fig. S4.1). This suggests the importance of regional dynamics for limpet co-occurrence and the potential importance of local dynamics not considered here, such as crabs and birds as additional predators. Variation in the environment $\left(\Delta_{i}^{E}\right)$, in particular, destabilizes limpet coexistence under low larval supplies enough to counteract the stabilizing effect of $\Delta_{i}^{E P}$. Under high larval supplies, a weak destabilizing effect of $\Delta_{i}^{E P}$ yields a negative growth rate when rare.

\section{Discussion}

While coexistence theory and food web theory examine similar core questions, they do so from traditionally disparate perspectives. Connections between the theories are becoming more common (e.g. Kuang \& Chesson (2008); Sommers \& Chesson (2019)), including recent developments that incorporate niche and fitness differences (Godoy et al., 2018). A unified framework that incorporates competition between species for shared resources or space together with effects of predation is necessary for gaining a synthetic understanding of how biodiversity is maintained. Our extension of Ellner et al. (2019), builds directly on the framework of modern coexistence theory (Chesson, 2000), including environmental fluctuations through time and space that can maintain coexistence among competitors via niche partitioning (Hallett et al., 2019; Letten et al., 2018). Critically, our extension of MCT for an environment-predation decomposition incorporates predation and fluctuations in predator abundances, allowing both bottom-up and top-down mechanisms to be incorporated simultaneously. The relative importance of both-as well as their interactions, as defined by the term $\Delta_{i}^{E P}$ — can be examined simultaneously, yielding an extension from the classic framework that allows for the examination of multiple fluctuations across trophic levels. In this study we focus on comparing bottom-up fluctuations in environmental conditions 
that alter prey mortality rates or larval supply rates and top-down fluctuations in predator abundances and predator larval supply rates.

Applying this approach, it becomes apparent that fluctuations in the environment or predator abundance are not always necessary for stabilizing species' growth rates when rare (e.g. Fig. 2B versus C), but rather individual species may preferentially require fluctuations. These results match insights from modern coexistence theory, even when focusing on a single trophic level in isolation (Hallett et al., 2019; Shoemaker \& Melbourne, 2016). Indeed, the superior competitor in the diamond model is only mildly affected by fluctuations in the environment and predator populations, while both environment and predator fluctuations increase the stability of the inferior competitor's $\left(C_{2}\right)$ growth rate when rare. Extending our approach to more complex food web dynamics highlights the importance of considering both food web topology and the strength of species interactions when quantifying coexistence and corresponding diversity expectations. For example, adding a new competitor to the classic, base diamond model yielded a destabilizing effect of relative nonlinearity in response to the environment in $C_{1}$ (Fig. 2) and a stabilizing effect in $C_{2}$. In general, the interactive effect of environmental fluctuations and predator abundances, $\Delta_{i}^{E P}$, appears to often be destabilizing (e.g. Fig. 2, S2.2, S2.3, and S2.4). While additional studies across systems and food web topology are necessary to determine the generality of this result, we hypothesize this trend may be general across food webs where competitors respond similarly to environmental fluctuations (e.g. Kuang \& Chesson (2010)). Environmental conditions that promote competitor growth will correspondingly yield increased predator abundances. Thus, the positive effects of environmental variation may be dampened by increased predation.

More generally, extending modern coexistence theory to examine multi-trophic systems yields key insights into the relative importance of top-down versus bottom-up forces in altering community composition and maintaining biodiversity (Gripenberg \& Roslin, 2007; Matson \& Hunter, 1992). In many systems, both factors work simultaneously to stabilize 
community dynamics. Our approach permits the partitioning of fluctuations in bottom-up $\left(\Delta_{i}^{E}\right)$ and top-down $\left(\Delta_{i}^{P}\right)$ dynamics to examine their relative importance, as well as considering their interaction $\left(\Delta_{i}^{E P}\right)$. For example, for intertidal barnacle species Balanus glandula and Chthamalus dalli, both top-down and bottom-up factors stabilize coexistence, although bottom-up factors appear to be slightly more important, at least according to our dynamical model formalization (Forde \& Doak, 2004). Empirically, in intertidal systems, the strength of bottom-up factors strongly covary with the strength of top-down processes (Menge et al., 1997). This covariation is destabilizing, as environmental conditions that promote barnacle growth also increase predator abundances. More generally, a similar approach could be applied across ecosystems to partition the relative importance of top-down and bottom-up effects operating simultaneously. For example, in grasslands, nutrient additions often decrease plant biodiversity while herbivores provide a counteracting effect, primarily by reducing light limitation (Borer et al., 2014). Furthermore, in the aquatic detritus-based ecosystems of carnivorous pitcher plants, nutrient additions tend to increase bacterial abundance but not biodiversity, while top predators increase bacterial biodiversity, likely through regulation of intermediate trophic levels (Kneitel \& Miller, 2002). We encourage future work that partitions coexistence in these different ecosystems, extending empirical applications beyond the rocky intertidal.

Our approach is general across both theoretical and empirical contexts; however, it requires a tight-coupling of demographic studies with interaction networks to yield dynamical models of both competitor and predator abundances. Measurements of demographic rates and food web interactions are usually made separately - often entire studies in their own rights (e.g. Dibner et al. (2019); Gripenberg et al. (2019)), and thus all the necessary information is difficult to obtain for many systems. As such, we encourage future work to examine both demographic rates along with trophic links and their corresponding strengths. Doing so will allow for quantifying coexistence and stability, along with the baseline structure of food 
webs (Pascual et al., 2006). In complex ecosystems, the number of equations and parameters quickly grow with the number of species, so simplifying into functional groups or exploring key species of interest may be necessary for computational tractability.

While here we focus on how the underlying structure of food webs and species' demography may promote coexistence, a similar approach could additionally incorporate behavioral dynamics. For example, recent work by Sommers and Chesson (2019) show that predator avoidance behavior by prey species can alter coexistence via changes in the importance of apparent competition relative to resource competition. If prey species partition resources, these behavioral changes tend to promote coexistence; when prey species instead partition predators, behavioral modifications undermine coexistence (Sommers \& Chesson, 2019). In multiple empirical systems, behavioral changes in prey species via fear-driven avoidance are similarly well documented, such as with brown anoles, green anoles, and curly-tailed lizards on tropical islands (Pringle et al., 2019). Additionally, predators have recently been shown to alter their behavior due to fear-driven avoidance of humans, which positively impacts prey species by increasing their foraging ability (Suraci et al., 2019). Other behaviors, such as omnivory, fundamentally change the topology and directional flow of food webs. Omnivory can both destabilize or stabilize a food web (Kratina et al., 2012), and presents a fascinating future direction. Partitioning the mechanistic effects of omnivory-including with multiple predator trophic levels - would yield insight into the potential stabilizing effects of complex food web topologies and energy flow on coexistence of prey species.

A further extension of this work could be examining coexistence under global change, with directional changes in environmental fluctuation (Usinowicz \& Levine, 2018). For instance, as temperature increases so do metabolic and encounter rates, which likely have important ramifications for coexistence, and in particular the contribution of relative nonlinearity in predation, $\Delta_{i}^{P}$ (Moya-Laraño et al., 2014). Additionally, this framework could be extended to examine eco-evolutionary dynamics. Classic single-trophic level applications of 
modern coexistence theory show the ability for stabilizing coexistence mechanisms to evolve, especially if one species has a greater evolutionary ability (Snyder \& Adler, 2011). In contrast, multiple species can co-evolve in a manner that erodes the importance of stabilizing coexistence mechanisms (Snyder \& Adler, 2011). Extensions examining phenotypic variation (Gibert \& DeLong, 2017) or eco-evolutionary dynamics in food webs may be critical, as both can drastically alter food web dynamics, links, and species' abundances, even under relatively small variation or selective pressures (Gibert \& Yeakel, 2019). Applications to eco-evolutionary food webs could provide insight into the evolutionary and environmental factors impacting species coexistence and community stability.

\section{Acknowledgements}

We thank Artemy Kolchinsky, Andy Rominger, and Margaret Siple for conversations in a Santa Fe Institute (SFI) Research Jam that led to the development of this project. We thank Dr. David Vasseur and two anonymous reviewers for suggestions that greatly improved the manuscript. This work was supported by a James S. McDonnell Foundation (JSMF)-SFI Postdoctoral Working Group grant to LGS and AKB (grant number: 357(14)). LGS, AKB, and LSB were supported by JSMF Postdoctoral Fellowships, grants \#220020513, \#220020478, and \#220020477. AIT was supported by the Omidyar Program at the SFI.

\section{Supporting Information}

Appendix S1: Derivation of Coexistence Mechanisms

Appendix S2: The Classic Diamond Model

Appendix S3: Expanding the Diamond Model for Additional Complexity

Appendix S4: Rocky Intertidal Food Web Case Study 


\section{References}

Abrams, P. A. (2009). When does greater mortality increase population size? The long history and diverse mechanisms underlying the hydra effect. Ecology Letters, 12, 462-474.

Ardia, D., Mullen, K. M., Peterson, B. G. \& Ulrich, J. (2016). DEoptim: Differential evolution in $\mathrm{R}$.

Barabás, G., D’Andrea, R. \& Stump, S. M. (2018). Chesson's coexistence theory. Ecological Monographs, 88, 277-303.

Berlow, E. L., Neutel, A.-M., Cohen, J. E., de Ruiter, P. C., Ebenman, B., Emmerson, M., Fox, J. W., Jansen, V. A. A., Iwan Jones, J., Kokkoris, G. D., Logofet, D. O., McKane, A. J., Montoya, J. M. \& Petchey, O. (2004). Interaction strengths in food webs: issues and opportunities. Journal of Animal Ecology, 73, 585-598.

Borer, E. T., Seabloom, E. W., Gruner, D. S., Harpole, W. S., Hillebrand, H., Lind, E. M., Adler, P. B., Alberti, J., Anderson, T. M., Bakker, J. D. et al. (2014). Herbivores and nutrients control grassland plant diversity via light limitation. Nature, 508, 517.

Brose, U. (2008). Complex food webs prevent competitive exclusion among producer species. Proceedings of the Royal Society B: Biological Sciences, 275, 2507-14.

Chesson, P. (2000). Mechanisms of maintenance of species diversity. Annual Review of Ecology and Systematics, 31, 343-358.

Chesson, P. \& Kuang, J. J. (2008). The interaction between predation and competition. Nature, 456, 235-238.

Cohen, J. E. \& Stephens, D. W. (1978). Food Webs and Niche Space. No. 11 in Monographs in Population Biology. ISBN 978-0-691-08202-8. 
This is an author-produced, peer-reviewed version of this article. The final, definitive version of this document can be found online at Ecology Letters, published by John Wiley \& Sons Ltd/CNRS. Copyright restrictions may apply. doi: 10.1111/ele.13482

Condit, R., Hubbell, S. P. \& Foster, R. B. (1995). Mortality rates of 205 neotropical tree and shrub species and the impact of a severe drought. Ecological monographs, 65, 419-439.

Connell, J. H. (1961). The influence of interspecific competition and other factors on the distribution of the barnacle Chthamalus stellatus. Ecology, 42, 710-723.

Connolly, S. R. \& Roughgarden, J. (1999). Theory of marine communities: competition, predation, and recruitment-dependent interaction strength. Ecological Monographs, 69, $277-296$.

Dayton, P. K. (1971). Competition, disturbance, and community organization: the provision and subsequent utilization of space in a rocky intertidal community. Ecological Monographs, 41, 351-389.

Dibner, R. R., Peterson, M. L., Louthan, A. M. \& Doak, D. F. (2019). Multiple mechanisms confer stability to isolated populations of a rare endemic plant. Ecological Monographs, 89, $\mathrm{e} 01360$.

Ellner, S. P., Snyder, R. E., Adler, P. B. \& Hooker, G. (2019). An expanded modern coexistence theory for empirical applications. Ecology Letters, 22, 3-18.

Forde, S. E. \& Doak, D. F. (2004). Multitrophic interactions mediate recruitment variability in a rocky intertidal community. Marine Ecology Progress Series, 275, 33-45.

Germain, R. M., Mayfield, M. M. \& Gilbert, B. (2018). The filtering metaphor revisited: competition and environment jointly structure invasibility and coexistence. Biology letters, $14,20180460$.

Gibert, J. P. \& DeLong, J. P. (2017). Phenotypic variation explains food web structural patterns. Proceedings of the National Academy of Sciences, 114, 11187-11192. 
Gibert, J. P. \& Yeakel, J. (2019). Eco-evolutionary origins of diverse abundance, biomass, and trophic structures in food webs. Frontiers in Ecology and Evolution, 7, 15.

Godoy, O., Bartomeus, I., Rohr, R. P. \& Saavedra, S. (2018). Towards the Integration of Niche and Network Theories. Trends in Ecology $\&$ Evolution, 33, 287-300.

Griffin, J. N., Byrnes, J. E. K. \& Cardinale, B. J. (2013). Effects of predator richness on prey suppression: a meta-analysis. Ecology, 94, 2180-2187.

Gripenberg, S., Basset, Y., Lewis, O. T., Terry, J. C. D., Wright, S. J., Simón, I., Fernández, D. C., Cedeño-Sanchez, M., Rivera, M., Barrios, H. et al. (2019). A highly resolved food web for insect seed predators in a species-rich tropical forest. Ecology Letters.

Gripenberg, S. \& Roslin, T. (2007). Up or down in space? uniting the bottom-up versus top-down paradigm and spatial ecology. Oikos, 116, 181-188.

Gurevitch, J., Morrison, J. A. \& Hedges, L. V. (2000). The interaction between competition and predation: a meta-analysis of field experiments. The American Naturalist, 155, 435453.

Hallett, L. M., Shoemaker, L. G., White, C. T. \& Suding, K. N. (2019). Rainfall variability maintains grass-forb species coexistence. Ecology Letters.

Holt, R. D. (1984). Spatial heterogeneity, indirect interactions, and the coexistence of prey species. The American Naturalist, 124, 377-406.

Holt, R. D., Grover, J. \& Tilman, D. (1994). Simple Rules for Interspecific Dominance in Systems with Exploitative and Apparent Competition. The American Naturalist, 144, $741-771$.

Ives, A. R., Cardinale, B. J. \& Snyder, W. E. (2005). A synthesis of subdisciplines: predatorprey interactions, and biodiversity and ecosystem functioning. Ecology Letters, 8, 102-116. 
Iwasa, Y. \& Roughgarden, J. (1986). Interspecific competition among metapopulations with space-limited subpopulations. Theoretical Population Biology, 30, 194-214.

Jabot, F. \& Bascompte, J. (2012). Bitrophic interactions shape biodiversity in space. Proceedings of the National Academy of Sciences, 201107004.

Klauschies, T. \& Gaedke, U. (2019). Predator-prey nutrient competition undermines predator coexistence. bioRxiv, 535195 .

Kneitel, J. M. \& Miller, T. E. (2002). Resource and top-predator regulation in the pitcher plant (sarracenia purpurea) inquiline community. Ecology, 83, 680-688.

Kraft, N. J., Godoy, O. \& Levine, J. M. (2015). Plant functional traits and the multidimensional nature of species coexistence. Proceedings of the National Academy of Sciences, $112,797-802$.

Kratina, P., LeCraw, R. M., Ingram, T. \& Anholt, B. R. (2012). Stability and persistence of food webs with omnivory: Is there a general pattern? Ecosphere, 3, art50.

Kuang, J. \& Chesson, P. (2008). PredationCompetition Interactions for Seasonally Recruiting Species. The American Naturalist, 171, E119-E133.

Kuang, J. J. \& Chesson, P. (2009). Coexistence of annual plants: Generalist seed predation weakens the storage effect. Ecology, 90, 170-182.

Kuang, J. J. \& Chesson, P. (2010). Interacting coexistence mechanisms in annual plant communities: Frequency-dependent predation and the storage effect. Theoretical Population Biology, 77, 56-70.

Letten, A. D., Dhami, M. K., Ke, P.-J. \& Fukami, T. (2018). Species coexistence through simultaneous fluctuation-dependent mechanisms. Proceedings of the National Academy of Sciences, 115, 6745-6750. 
This is an author-produced, peer-reviewed version of this article. The final, definitive version of this document can be found online at Ecology Letters, published by John Wiley \& Sons Ltd/CNRS. Copyright restrictions may apply. doi: 10.1111/ele.13482

Levins, R. \& Culver, D. (1971). Regional Coexistence of Species and Competition between Rare Species. Proceedings of the National Academy of Sciences, 68, 1246-1248.

Matson, P. A. \& Hunter, M. D. (1992). Special feature: The relative contributions to topdown and bottom-up forces in population and community ecology. Ecology, 73, 723-723.

May, R. M. (1973). Qualitative stability in model ecosystems. Ecology, 54, 638-641.

McCann, K., Hastings, A. \& Huxel, G. R. (1998). Weak trophic interactions and the balance of nature. Nature, 395, 794-798.

McCann, K. S. (2011). Food Webs. No. 50 in Monographs in Population Biology. Princeton University Press. ISBN 978-1-4008-4068-7.

McPeek, M. A. (2019). Limiting Similarity? The Ecological Dynamics of Natural Selection among Resources and Consumers Caused by Both Apparent and Resource Competition. The American Naturalist, E000-E000.

Menge, B. A. (2000). Recruitment vs. post recruitment processes as determinants of barnacle population abundance. Ecological Monographs, 70, 265-288.

Menge, B. A., Daley, B. A., Wheeler, P. A., Dahlhoff, E. P., Sanford, E. \& Strub, P. T. (1997). Benthic-pelagic links and rocky intertidal communities: Bottom-up effects on topdown control? Proceedings of the National Academy of Sciences of the United States of America, 94, 14530-14535.

Moya-Laraño, J., Bilbao-Castro, J. R., Barrionuevo, G., Ruiz-Lupión, D., Casado, L. G., Montserrat, M., Melián, C. J. \& Magalhães, S. (2014). Eco-evolutionary spatial dynamics: rapid evolution and isolation explain food web persistence. In: Advances in ecological research, vol. 50. Elsevier, pp. 75-143. 
Paine, R. T. (1966). Food web complexity and species diversity. The American Naturalist, $100,65-75$.

Paine, R. T. (1969). A note on trophic complexity and community stability. The American Naturalist, 103, 91-93.

Pascual, M., Dunne, J. A. et al. (2006). Ecological networks: linking structure to dynamics in food webs. Oxford University Press.

Pringle, R. M., Kartzinel, T. R., Palmer, T. M., Thurman, T. J., Fox-Dobbs, K., Xu, C. C., Hutchinson, M. C., Coverdale, T. C., Daskin, J. H., Evangelista, D. A. et al. (2019). Predator-induced collapse of niche structure and species coexistence. Nature, 570, 58.

R Core Team (2019). R: A Language and Environment for Statistical Computing. R Foundation for Statistical Computing, Vienna, Austria. URL https://www.R-project.org/.

Ruokolainen, L., Ranta, E., Kaitala, V. \& Fowler, M. S. (2009). Community stability under different correlation structures of species environmental responses. Journal of theoretical biology, 261, 379-387.

Saleem, M., Fetzer, I., Dormann, C. F., Harms, H. \& Chatzinotas, A. (2012). Predator richness increases the effect of prey diversity on prey yield. Nature Communications, 3, 1305.

Shinen, J. L. \& Navarrete, S. A. (2014). Lottery coexistence on rocky shores: weak niche differentiation or equal competitors engaged in neutral dynamics? The American Naturalist, 183.

Shoemaker, L. G. \& Melbourne, B. A. (2016). Linking metacommunity paradigms to spatial coexistence mechanisms. Ecology, 97, 2436-2446. 
Shoemaker, L. G., Sullivan, L. L., Donohue, I., Cabral, J. S., Williams, R. J., Mayfield, M. M., Chase, J. M., Chu, C., Harpole, W. S., Huth, A. et al. (2019). Integrating the underlying structure of stochasticity into community ecology. Ecology.

Snyder, R. E. \& Adler, P. B. (2011). Coexistence and coevolution in fluctuating environments: can the storage effect evolve? The American Naturalist, 178, E76-E84.

Sommers, P. \& Chesson, P. (2019). Effects of predator avoidance behavior on the coexistence of competing prey. The American Naturalist, 193, E132-E148.

Suraci, J. P., Clinchy, M., Zanette, L. Y. \& Wilmers, C. C. (2019). Fear of humans as apex predators has landscape-scale impacts from mountain lions to mice. Ecology letters, Early View.

Terborgh, J. W. (2015). Toward a trophic theory of species diversity. Proceedings of the National Academy of Sciences, 112, 11415-11422.

Tilman, D. (1982). Resource Competition and Community Structure. No. 17 in Monographs in Population Biology. Princeton University Press. ISBN 978-0-691-08302-5.

Usinowicz, J. \& Levine, J. M. (2018). Species persistence under climate change: a geographical scale coexistence problem. Ecology letters, 21, 1589-1603.

Vasseur, D. A. \& Fox, J. W. (2007). Environmental fluctuations can stabilize food web dynamics by increasing synchrony. Ecology Letters, 10, 1066-1074.

Williams, R. J. \& Martinez, N. D. (2000). Simple rules yield complex food webs. Nature, 404, 180-183.

Yu, D. W. \& Wilson, H. B. (2001). The competition-colonization trade-off is dead; long live the competition-colonization trade-off. The American Naturalist, 158, 49-63. 


\begin{tabular}{|c|c|c|}
\hline Parameter & Description & Value \\
\hline \multicolumn{3}{|c|}{2 Competitors, 1 Predator Diamond Model } \\
\hline$r$ & resource density independent growth rate & 1.0 \\
\hline$K$ & resource carrying capacity & 1.0 \\
\hline$J_{C_{1}}$ & competitor 1 resource intake rate & 0.8036 \\
\hline$J_{C_{2}}$ & competitor 2 resource intake rate & 0.7 \\
\hline$J_{P}$ & predator intake rate & 0.4 \\
\hline$M_{C_{1,0}}$ & competitor 1 beginning mortality rate & 0.4 \\
\hline$M_{C_{2,0}}$ & competitor 2 beginning mortality rate & 0.2 \\
\hline$M_{P}$ & predator mortality rate & 0.08 \\
\hline$R_{0_{1}}$ & half saturation constant; resource use by competitor 1 & 0.16129 \\
\hline$R_{0_{2}}$ & half saturation constant; resource use by competitor 2 & 0.9 \\
\hline$C_{0}$ & predator half saturation constant & 0.5 \\
\hline$\Omega_{P C_{1}}$ & predator preference for competitor 1 & 0.92 \\
\hline$\Omega_{C_{1 R}}$ & competitor 1 preference for the resource & 1.0 \\
\hline$\Omega_{C_{2 R}}$ & competitor 2 preference for the resource & 0.98 \\
\hline \multicolumn{3}{|c|}{3 Competitors, 1 Predator Extended Model } \\
\hline$J_{C_{3}}$ & competitor 3 resource intake rate & $0.862126,0.335544$ \\
\hline$M_{C_{3,0}}$ & competitor 3 beginning mortality rate & $0.262571,0.149855$ \\
\hline$R_{0_{3}}$ & half saturation constant; resource use by competitor 3 & $0.227814,0.204875$ \\
\hline$\Omega_{P C_{1}}$ & predator preference coefficient for competitor 1 & $\mathbf{0 . 8 8 1 3 9 7 ,} 0.869924$ \\
\hline$\Omega_{P C_{2}}$ & predator preference coefficient for competitor 2 & $0.065556,0.079664$ \\
\hline$\Omega_{C_{3 R}}$ & competitor 3 preference for the resource & $\mathbf{0 . 5 3 3 1 6 4 , 0 . 7 3 9 0 8 7}$ \\
\hline \multicolumn{3}{|c|}{3 Competitors, 2 Predators Extended Model } \\
\hline$M_{P_{2}}$ & predator 2 mortality rate & 0.0700288 \\
\hline$J_{P_{2}}$ & predator 2 intake rate & 0.407796 \\
\hline$\Omega_{P_{2} C_{1}}$ & predator 2 preference coefficient for $C_{1}$ & 0.398767 \\
\hline$\Omega_{P_{2} C_{2}}$ & predator 2 preference coefficient for $C_{2}$ & 0.129095 \\
\hline$C_{0_{P 2}}$ & predator 2 half saturation constant & 0.885837 \\
\hline
\end{tabular}

Table 1: Parameters in the diamond model and its extensions to incorporate additional food web complexity. Parameterization of the classic diamond model matches that from Vasseur \& Fox (2007). The mechanistic decomposition of the 2 competitors, 1 predator system is shown in Fig. 2B,C. The bold values from the 3 competitors, 1 predator extended model are those of replicate 1, shown in Fig. 2E-G. The values of replicate 2 are given beside the estimates from replicate 1 and are shown in Fig. S3.7I-K. The bold parameters are subsequently used for the 2 predator extension (Fig. 2I-K). 


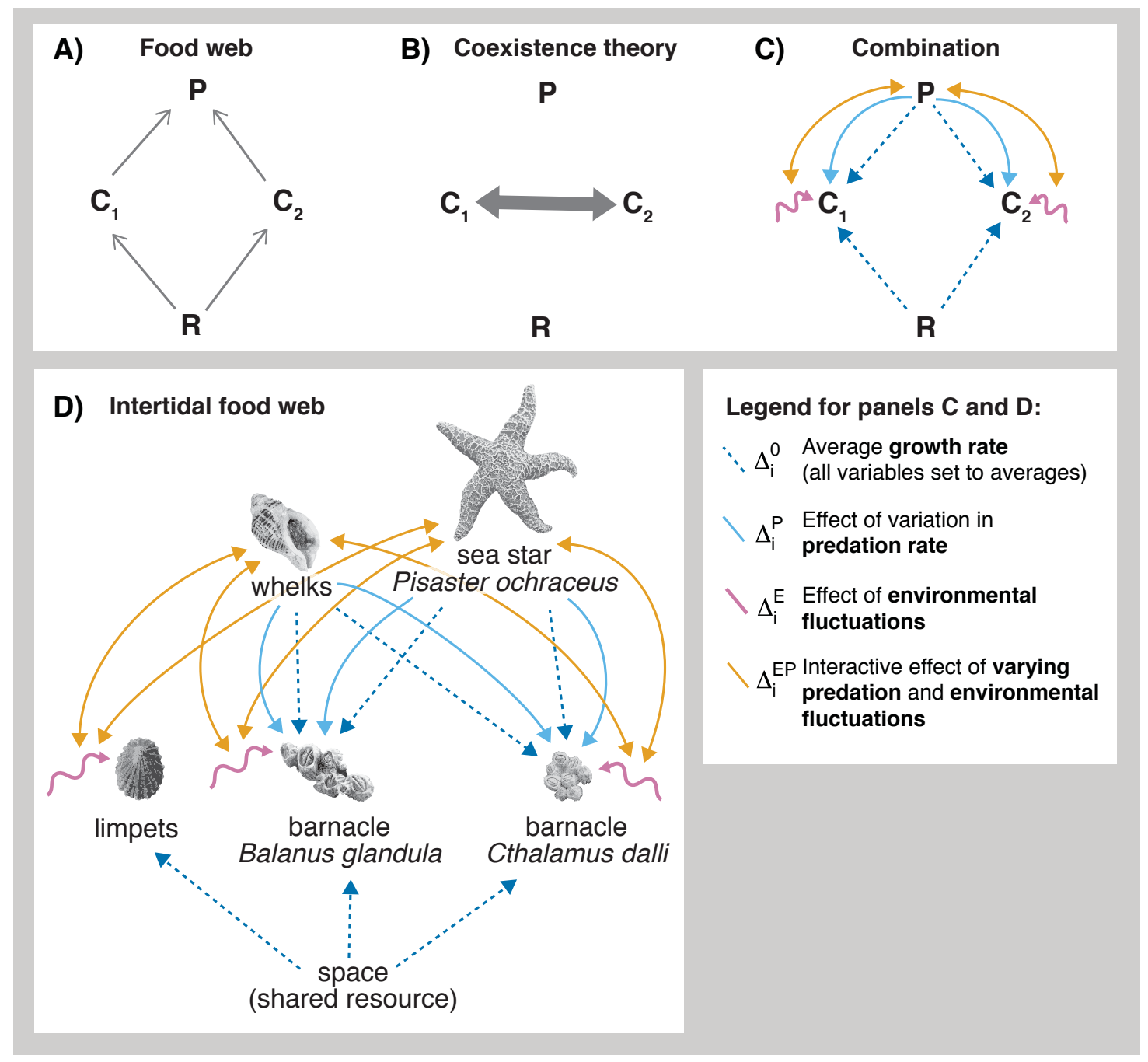

Figure 1: Conceptual diamond model (A-C) and the rocky intertidal food web (D). (A) Food webs generally have links connecting trophic levels where directed links (arrows), point in the direction of energy and matter flow, that is, from resources to competitors (C1 and C2 share a resource, $\mathrm{R}$, and are consumed by preadator, $\mathrm{P}$ ). (B) Coexistence theory generally addresses only competition at one trophic level-here illustrated with a wide, double-pointed arrow - integrating consumption of a shared resource into an interaction coefficient. (C) In the framework proposed here, we combine food webs and coexistence theory, using the mutual invasion criterion and an approach that allows for coexistence to be partitioned into species' average growth rates $\Delta_{i}^{0}$, predation variability $\Delta_{i}^{P}$, environmental fluctuations $\Delta_{i}^{E}$, and the interaction between predation and environmental fluctuations $\Delta_{i}^{E P}$ (Box 1). Here, we use arrows to represent the effects of variation in predation and environmental fluctuations (and their interaction) on focal species. (D) In the rocky intertidal community of the Northeast Pacific Ocean, barnacles and limpets compete for space, and environmental fluctuations (pink) lead to variation in their larval supply. Sea stars and whelks influence barnacle dynamics through predation (blue). Limpets, however, are not consumed. 

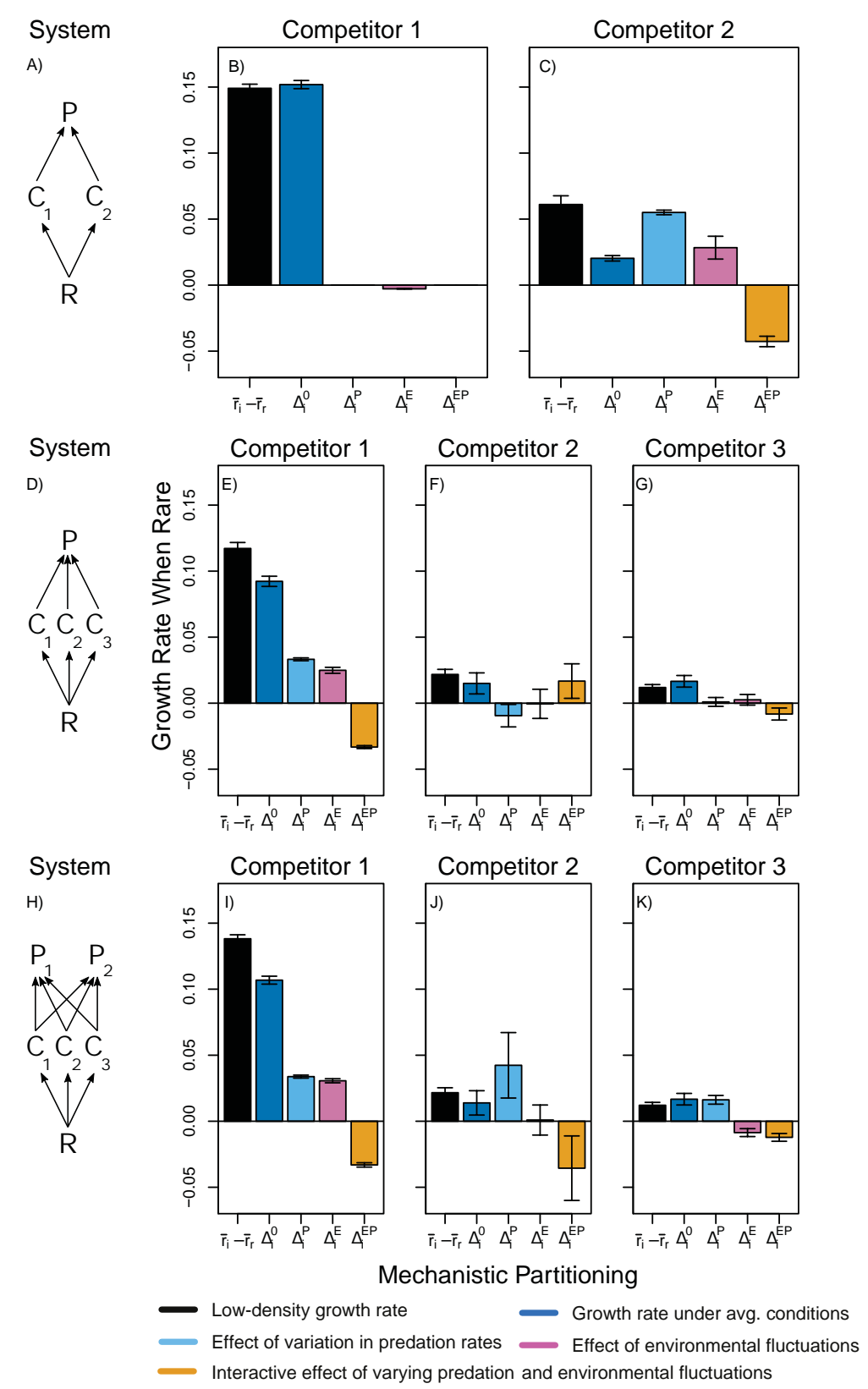

Figure 2: Decomposition of three food webs, showing overall growth rate $\bar{r}_{i}-\overline{r_{r}}$, the growth rate with no environmental or predator fluctuations $\left(\Delta_{i}^{0}\right)$, the effect of fluctuations in predator abundance $\left(\Delta_{i}^{P}\right)$, the effect of fluctuations in environmental conditions $\left(\Delta_{i}^{E}\right)$, and their combined effects $\left(\Delta_{i}^{E P}\right)$. (A) Classic food web diamond model. (B-C) Mechanistic partitioning for each of the competitors in diamond food web containing two competitors. (D) An expansion of the diamond model that includes a third competitor. (E-G) Mechanistic partitioning for each of the competitors in (D). (H) A further expansion of the diamond model that includes a third competitor and a second predator. (I-K) Mechanistic partitioning for each of the competitors in $(\mathrm{H})$. Results show mean and standard deviation across 500 runs with no cross-correlation of environmental fluctuations between species $(\rho=0)$. 


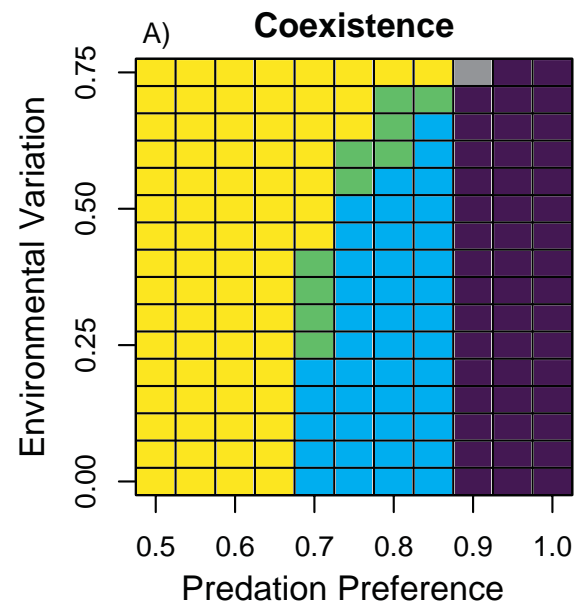

$\square$ Species 1 Wins

Species 2 Wins

Coexistence

Species 1 or 2 Wins

Coexistence or Species 1 Wins
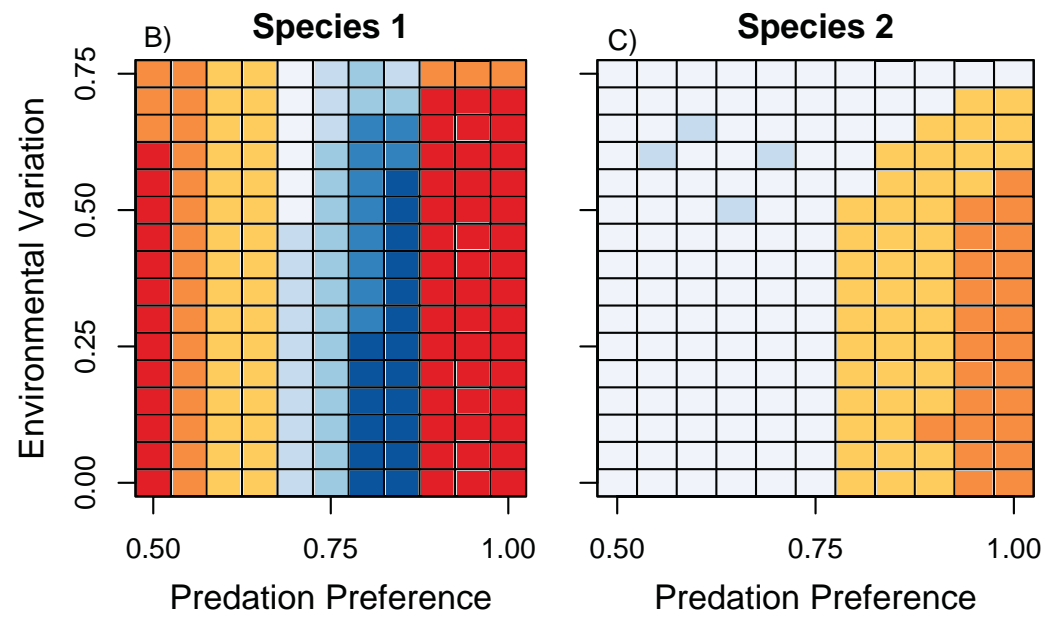

Growth Rate

When Rare

$-0.15$

0.10

$-0.05$

$-0.00$

$-0.05$

$-0.10$

$-0.15$

$-0.20$

Figure 3: (A) Comparison of the role of predation preference (i.e. top down effects) versus variation in the environment (i.e. bottom up effects) on coexistence of both competitors in the diamond model. Coexistence requires that both species' growth rates when rare are positive (panels B, C; orange and red colors). For species 1, increasing predation preference decreases its growth rate when rare initially, but then allows for coexistence via oscillatory dynamics. For species 2, increasing the predation preference for species 1 increases species 2 's growth rate when rare. Results are shown for 1,760 runs (10 runs per each parameter combination). 

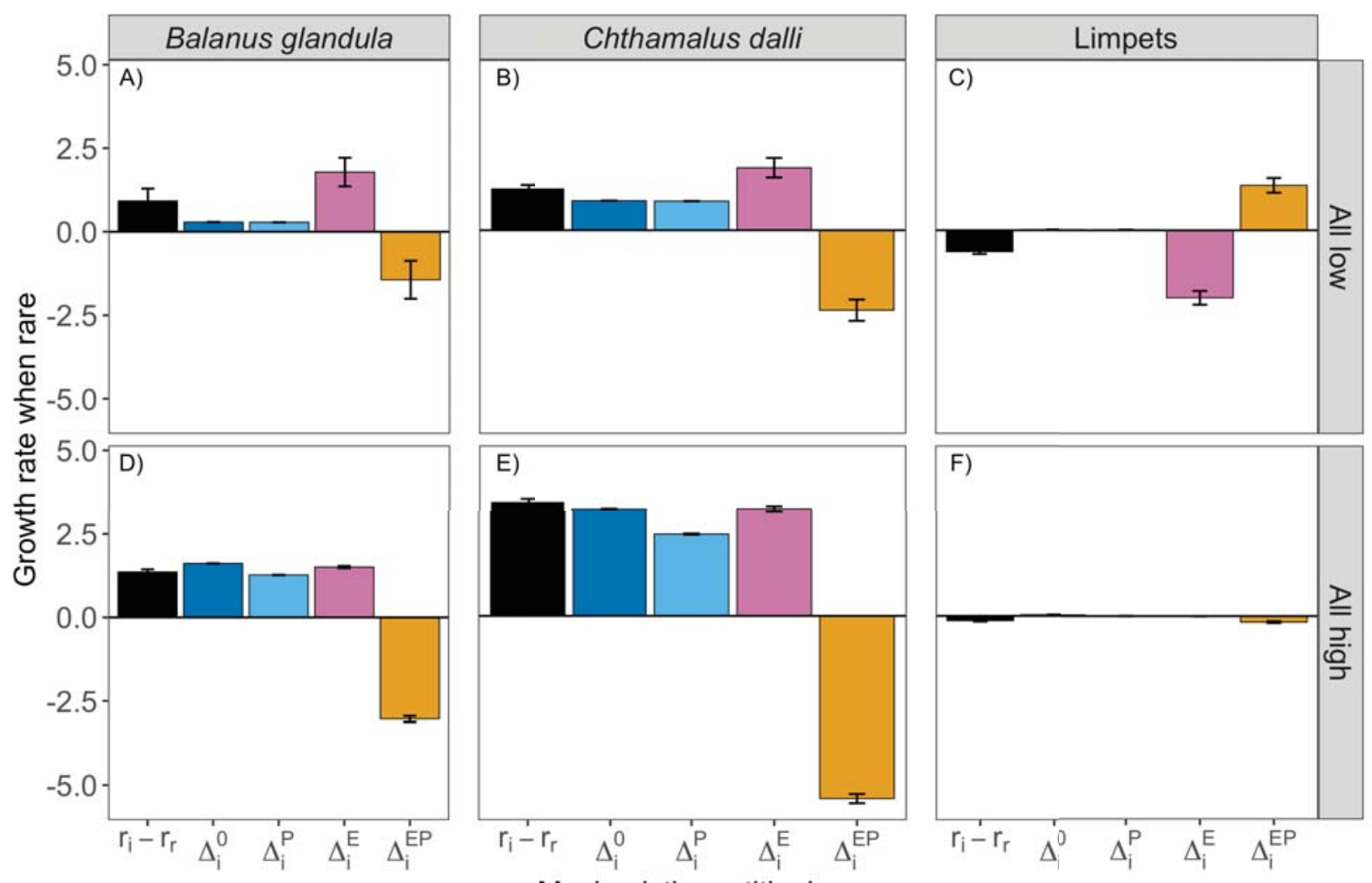

Mechanistic partitioning

\section{- Low-density growth rate}

Growth rate under avg. conditions

Effect of variation in predation rates

Effect of environmental fluctuations

Interactive effect of varying predation and environmental fluctuations

Figure 4: Application of coexistence partitioning to the empirical rocky intertidal foodweb, comparing two levels of larval supply (high and low). Results show the mean and standard error for 500 replicates, each run for 100 years. Both barnacles coexist, despite a destabilizing effect of the interaction between environment and predator fluctuations $\left(\Delta_{i}^{E P}\right)$. Limpets exhibit a slight negative growth rate when rare, suggesting competitive exclusion. Note that neither predators consume limpets, thus the effect of $\Delta_{i}^{E P}$ on limpet coexistence strength is an indirect effect mediated by predation on barnacles (see Fig. 1D). Additional larval supply scenarios are presented in Fig. S4.1 


\section{Box 1. Mechanistic Partitioning of Coexistence Mechanisms}

Central to this study, we examine the relative importance of environmental fluctuations versus fluctuations in predator abundances for coexistence, building on the classic framework of MCT (Chesson, 2000; Barabás et al., 2018) and recent advances for flexible applications using a simulation-based approach (Ellner et al., 2019). We quantify the contribution of fluctuations in top-down (predation) and bottom-up (environmental) processes on species persistence. Here, we give an overview of the decomposition; a full derivation is provided in Supplement 1.

Coexistence occurs in the MCT framework if each species when rare (termed the invader due to its low-density) has a demographic advantage over the other resident species at their steady-state abundances. This advantage buffers the invader species from extinction. The mathematical quantification of this process is termed the growth rate when rare and is calculated as the difference in growth rates between the focal invader species $i$ at low-density and the resident species, $r: \bar{r}_{i}-\bar{r}_{r}$. If $\bar{r}_{i}-\bar{r}_{r}>0$ for all species as the invader, then stable coexistence occurs. Alternatively, if a species as the invader exhibits $\bar{r}_{i}-\bar{r}_{r}<0$, we predict the species would be driven to competitive exclusion.

Each species' growth rate when rare can be decomposed into its mechanistic contributions. Analytical MCT mechanisms follow an environment-competition decomposition quantifying small variance approximations which yields the classic mechanisms the storage effect, relative nonlinearity in competition, and fitness-density covariance (for spatial variabilitiy) (Chesson, 2000; Barabás et al., 2018). However, the flexibility of the Ellner et al. (2019) framework allows for alternative decompositions depending on the features of interest. Here, we implement a predator abundance versus environmental fluctuation decomposition (see Supplement 1 for derivation), yielding the full 
decomposition:

$$
\bar{r}_{i}-\bar{r}_{r}=\Delta_{i}^{0}+\Delta_{i}^{P}+\Delta_{i}^{E}+\Delta_{i}^{E P}
$$

The mechanisms of this decomposition are as follows:

1. $\Delta_{i}^{0}$ : growth rate under average conditions quantifies the difference in invader and resident growth rates at mean environmental conditions and predator abundances. It reflects the growth rate when rare if no top-down or bottom-up fluctuations are present (Fig. 1C, dashed blue; note the change in arrow directions compared to $1 \mathrm{~A}$ which denotes the focus on the competitor trophic level).

2. $\Delta_{i}^{P}$ : relative nonlinearity in predation quantifies the main effects of variability in predator abundance. It is calculated as the difference between (1) growth rate when predator abundance varies but the environment is constant at its mean conditions and (2) the growth rate under average conditions. Following the nomenclature of Ellner et al. (2019) we refer to main-effect terms as "nonlinearity" as, in this case, any difference from zero results from a nonlinear response of growth rate to predator fluctuations above and below the mean (Fig. 1C, light blue).

3. $\Delta_{i}^{E}$ : relative nonlinearity in response to the environment quantifies the main effects of variability in environmental conditions. It is calculated as the difference between (1) growth rates when the environment varies but predator abundances are held constant its mean and (2) the growth rate under average conditions (Fig. $1 \mathrm{C}$, pink).

4. $\Delta_{i}^{E P}$ : the interactive effect of fluctuations in predation and the environment accounts for the additional change in growth rates when both factors vary, be- 
yond the contribution of each effect varying on its own $\left(\Delta_{i}^{P}\right.$ and $\left.\Delta_{i}^{E}\right)($ Fig. 1C, orange, note the double-headed arrow denoting the interaction between predators $P$ and environmental fluctuations, pink). This term includes the effect of uncorrelated joint variation and additional effects of correlation between environmental conditions and predator abundances. We note that under a classic analytical environment-competition MCT decomposition, this term incorporates the classic storage effect (Ellner et al., 2019), however we avoid this terminology here due to differences in interpretation with a predator versus competition decomposition.

By simulating different scenarios where we allow predator abundances, environmental conditions, both, or neither to vary, we can calculate the contribution of each mechanism to each species' growth rate when rare. Mechanisms may have minimal effects, a destabilizing effect (e.g. a negative contribution to a species' growth rate when rare, as seen with $\Delta_{i}^{E}$ for Limpets, Fig. $4 \mathrm{C}$ ), or a stabilizing effect (e.g. a positive contribution to a species' growth rate when rare, as seen with $\Delta_{i}^{E}$ for Balanus, Fig. 4A) on coexistence. 


\title{
Quantifying the relative importance of variation in predation and the environment for species coexistence

\author{
Derivation of Coexistence Mechanisms: Supplement 1
}

\author{
Lauren G. Shoemaker, Allison K. Barner, Leonora S. Bittleston, and Ashley I. Teufel
}

\section{Derivation}

Following classic MCT, we test for stable coexistence using the mutual invasion criterion, which requires that each species can invade when all other species in the food web (minus the invader) are at their steady state abundance distributions. We apply the invader-resident comparison, where we examine the invader's growth rate when rare, subtracting out the resident-competitors' average growth rates (Barabás et al., 2018; Ellner et al., 2019). In this approach, $\bar{r}_{i}-\bar{r}_{r} \approx \bar{r}_{i}$ (where $i$ denotes the invader and $r$ denotes the resident) since the residents' average growth rates will be 0 as they are at their steady state distributions and the invader is at such low density that interspecific competition is minimal. We incorporate the invader-resident comparison, however, as coexistence can be fostered by mechanisms that either help the invader or hinder the resident. Stable coexistence requires that $\bar{r}_{i}-\bar{r}_{r}>0$ for all species, where $r_{j}=\ln \frac{N_{j, t+1}}{N_{j, t}}$ for a species $j$.

The average population growth rate of each species, $j$, through time depends on both environmental fluctuations $(E(t))$ and fluctuations in predator abundances $(P(t))$, such that

$$
\bar{r}_{j}=\frac{1}{T} \sum_{t=1}^{T} r_{j}(E(t), P(t)) .
$$

On average, a population increases if $\bar{r}_{j}>0$. Critically, this formalization allows us to decompose growth rates into their mechanistic components. Following Ellner et al. 2019, we for each species we define the following terms:

$$
\begin{gathered}
\epsilon_{j}^{0}=r_{j}(\bar{E}, \bar{P}), \\
\epsilon_{j}^{E}=r_{j}(E(t), \bar{P})-\epsilon_{j}^{0}, \\
\epsilon_{j}^{P}=r_{j}(\bar{E}, P(t))-\epsilon_{j}^{0}
\end{gathered}
$$

and

$$
\epsilon_{j}^{E P}=r_{j}(E(t), P(t))-\left[\epsilon_{j}^{0}+\epsilon_{j}^{E}+\epsilon_{j}^{P}\right]
$$

$\epsilon_{j}^{0}$ is the population growth rate when the environment and predator abundances are constant at their means, $\epsilon_{j}^{E}$ is the main effect of the environment varying around its mean, $\epsilon_{j}^{P}$ is the main effect of predator abundance varying around its mean, and the term $\epsilon_{j}^{E P}$ accounts for the fact that having variability in both the environment and predator abundances will not equal the sum of the main effects.

Following Ellner et al. (2019), we find that

$$
r_{j}(E(t), P(t))=\epsilon_{j}^{0}+\epsilon_{j}^{E}(E)+\epsilon_{j}^{P}(P)+\epsilon_{j}^{E P}(E, P)
$$

which we can average to determine that:

$$
\bar{r}_{j}=\epsilon_{j}^{0}+\bar{\epsilon}_{j}^{E}+\bar{\epsilon}_{j}^{P}+\bar{\epsilon}_{j}^{E P}
$$

we use Equation S1.7 to compute the invader-resident comparison, where we compare $\bar{r}_{i}-\bar{r}$. For the invader-resident comparison, 


$$
\bar{r}_{i}=\bar{r}_{i}-\bar{r}_{r}=\Delta_{i}^{0}+\Delta_{i}^{E}+\Delta_{i}^{P}+\Delta_{i}^{E P}
$$

where $\Delta_{i}$ is the invader-resident difference between corresponding terms. For example, $\Delta_{i}^{0}=\epsilon_{i}^{0}-\epsilon_{r}^{0}$. The full derivation with further explanation can be found in Ellner et al. 2019. For scenarios with multiple resident competitor species (i.e. the expanded diamond model and the intertidal food web model), we weight all residents equally. While there are multiple choices for resident weighting in invader-resident comparisons (e.g. Eqn 20 from Ellner et al. 2019), we chose to weight residents equally for two reasons. First, it simplifies the interpretation compared to a pairwise comparison with each resident. Second, the classic weighting scaling factors (Chesson, 1994, 2000) do not exist or are not unique in many models. As such, we follow the approach advocated for in Ellner et al. 2019 and use the equal weighting. For example, under this weighting, $\Delta_{i}^{0}=\epsilon_{i}^{0}-\frac{1}{S-1} \sum_{r \neq i} \bar{\epsilon}_{r}^{0}$.

Equation S1.8 provides the full decomposition used throughout this study. Here, $\Delta_{i}^{0}$, examines species' ability to invade when rare if both the environment and predator abundances are constant at their means. The second term, $\Delta_{i}^{E}$, is nonlinearity in response to the environment, and it quantifies the effect of variability in environmental conditions. This directly affects competitor mortality rates in the classic diamond model and the expanded diamond model and larval supply rates in the rocky intertidal food web model. Intuitively, nonlinearity in response to the environment can stabilize a species' growth rate when rare if the positive effects of "good" environmental years are larger in magnitude than the negative effects of "bad" environmental years. Similarly, $\Delta_{i}^{P}$ is nonlinearity in predation and is stabilizing if the magnitude of consumptive effects at high predator abundances are less than population gains at low predator abundance (i.e. via saturating consumption). Finally, $\Delta_{i}^{E P}$ quantifies their interactive effects not accounted for by each main effect in isolation.

\section{Applications}

We first apply the above decomposition to the classic diamond model and the expanded diamond model. When calculating each mechanism, we ran the resident community for 5000 time steps. We used the first 2500 as a "burn-in" to remove any potential effect of starting conditions and defined the last 2500 time steps as the steady-state distribution. For each time point $t$ in the last 2500 time steps, we calculated the invader's growth rate when rare and the resident competitors' growth rates with an invader abundance of 0.001 . The diamond model exhibits between time-point variability, where quasiperiodic chaos occurs in the absence of the second competitor, $C_{2}$. Calculation of coexistence is therefore the average invasion growth rate $\bar{r}_{i}-\bar{r}_{r}$ from timepoints 2500 to 5000 . The strength of each mechanism is averaged in the same manner.

For the rocky intertidal food web, we ran the resident community for 100 years (1200 time steps in the model). Similarly to the diamond model, we used the first half as a "burn-in" to remove potential effects of starting conditions and defined the last 50 years as the steady-state distribution. We calculated the mutual invasion criterion with an invader abundance of 1 individual.

All code is available in our supplementary material.

\section{References}

Barabás, G., D’Andrea, R. \& Stump, S. M. (2018). Chesson's coexistence theory. Ecological Monographs, 88, 277-303.

Chesson, P. (1994). Multispecies competition in variable environments. Theoretical population biology, 45, $227-276$.

Chesson, P. (2000). Mechanisms of maintenance of species diversity. Annual Review of Ecology and Systematics, 31, 343-358.

Ellner, S. P., Snyder, R. E., Adler, P. B. \& Hooker, G. (2019). An expanded modern coexistence theory for empirical applications. Ecology Letters, 22, 3-18. 


\title{
Quantifying the relative importance of variation in predation and the environment for species coexistence
}

\section{The Classic Diamond Model: Supplement 2}

\author{
Lauren G. Shoemaker, Allison K. Barner, Leonora S. Bittleston, and Ashley I. Teufel
}

\section{Coexistence With No Environmental Variation}

As an intermediate step for analyzing the classic diamond model, below we should results for the model with no environmental variation ( $\sigma=0.0$; Fig. S2.1). Here, we see that the superior competitor's positive growth rate when rare (black bar) comes entirely from $\Delta_{i}^{0}$-it's growth rate under average conditions with no environmental or predator fluctuations. The inferior competitor maintains a positive growth rate when rare due to a slight contribution from $\Delta_{i}^{0}$, and a larger contribution from $\Delta_{i}^{P}$, as cyclic dynamics and relative nonlinearity help stabilize coexistence for $C_{2}$. Results show the average strength of each mechanism from timepoints 2500 to 5000 (as detailed in Eqn S1.1). No between-run variation occurs in the absence of environmental fluctuations.

\section{Altering Cross-Correlation of Environmental Variation and its Strength}

While altering the cross-correlation of environmental variation between species $(\rho)$ had little effect on the fluctuation dependent coexistence mechanisms (Figs. S2.2, S2.3), increasing the strength of environmental fluctuations $(\sigma)$ significantly changed the contributions of multiple coexistence mechanisms (Fig. S2.4). Increasing the strength of environmental fluctuations caused the strength of the growth rate when rare to decrease for the inferior competitor $\left(C_{2}\right)$. However, both relative nonlinearity in response to the environmental and relative nonlinearity in predation increased, further stabilizing coexistence with increasing environmental fluctuations. In comparison, their interactive effect became more destabilizing (i.e. negative). The superior competitor, however, was only minimally affected by changes in environmental fluctuations.

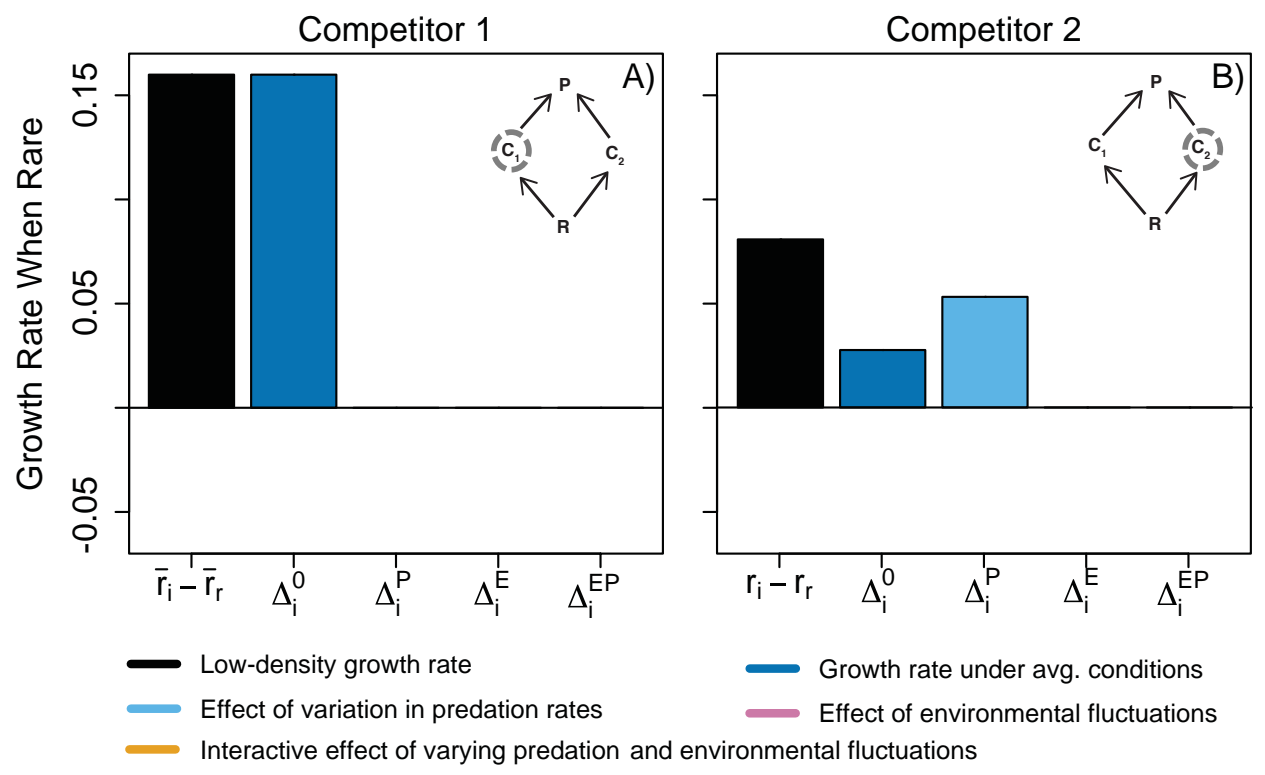

Figure S2.1: Coexistence under no environmental variation. Comparison to Fig. 2B and C, except with no environmental variation $(\sigma=0.0)$. No between run variation occurs in the absence of environmental fluctuations, explaining the lack of error bars. 


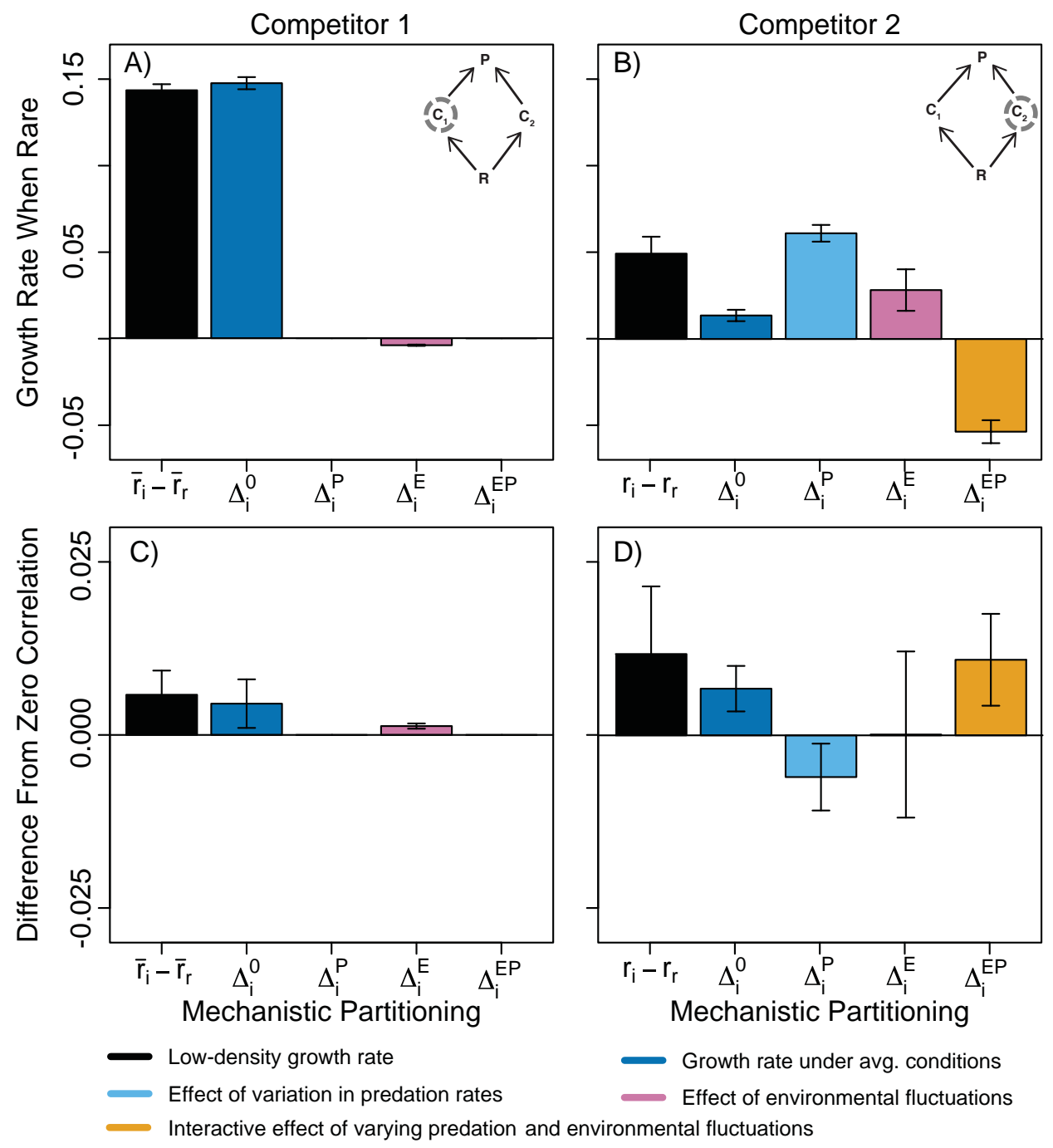

Figure S2.2: Positive cross correlation comparison to Fig. 2B,C in the main text. Examining the role of predation preference (i.e. top down effects) versus variation in the environment (i.e. bottom up effects) on coexistence of both competitors in the diamond model. Coexistence requires that both species' low-density growth rates are positive (panels A, B; black). Model parameters are the same as in Table 1, except with positive cross-correlation of environmental fluctuations between species $(\rho=0.75)$. The difference in strength of mechanisms between scenarios with no cross correlation (Fig. 3; $\rho=0.00$ ) are shown in panels $\mathrm{C}$ and D. Results are shown for 500 runs with error bars denoting standard deviation. 
This is an author-produced, peer-reviewed version of this article. The final, definitive version of this document can be found online at Ecology Letters, published by John Wiley \& Sons Ltd/CNRS. Copyright restrictions may apply. doi: 10.1111/ele.13482
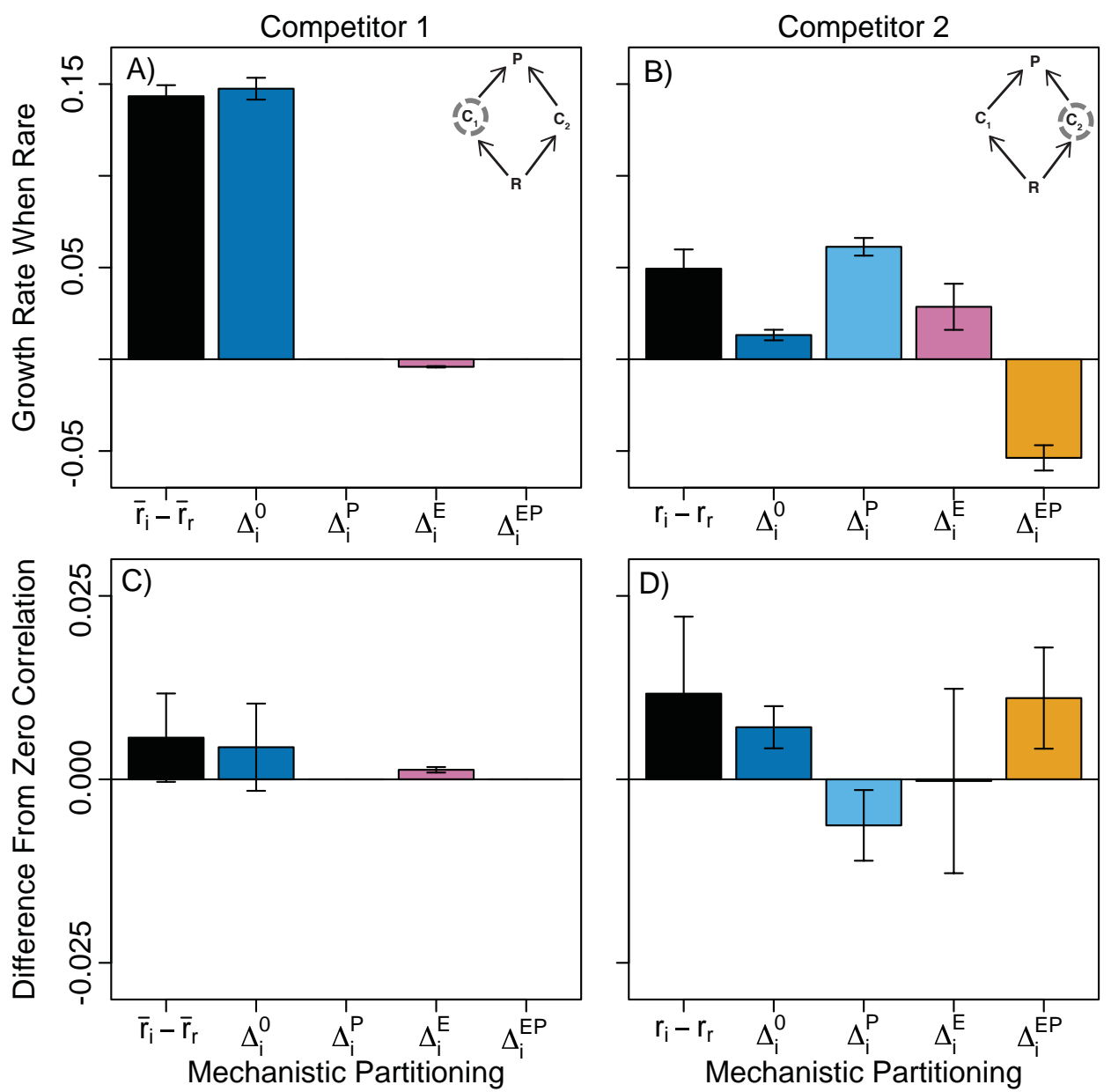

\section{Mechanistic Partitioning}

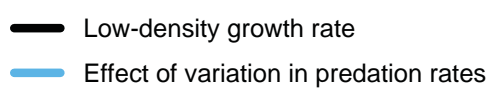

Growth rate under avg. conditions

Effect of environmental fluctuations

Interactive effect of varying predation and environmental fluctuations

Figure S2.3: Negative cross correlation comparison to Fig. 2B,C in the main text. Examining the role of predation preference (i.e. top down effects) versus variation in the environment (i.e. bottom up effects) on coexistence of both competitors in the diamond model. Coexistence requires that both species' low-density growth rates are positive (panels A, B; black). Model parameters are the same as in Table 1, except with negative cross-correlation of environmental fluctuations between species $(\rho=-0.75)$. The difference in strength of mechanisms between scenarios with no cross correlation (Fig. 3; $\rho=0.00$ ) are shown in panels $\mathrm{C}$ and D. Results are shown for 500 runs with error bars denoting standard deviation. 


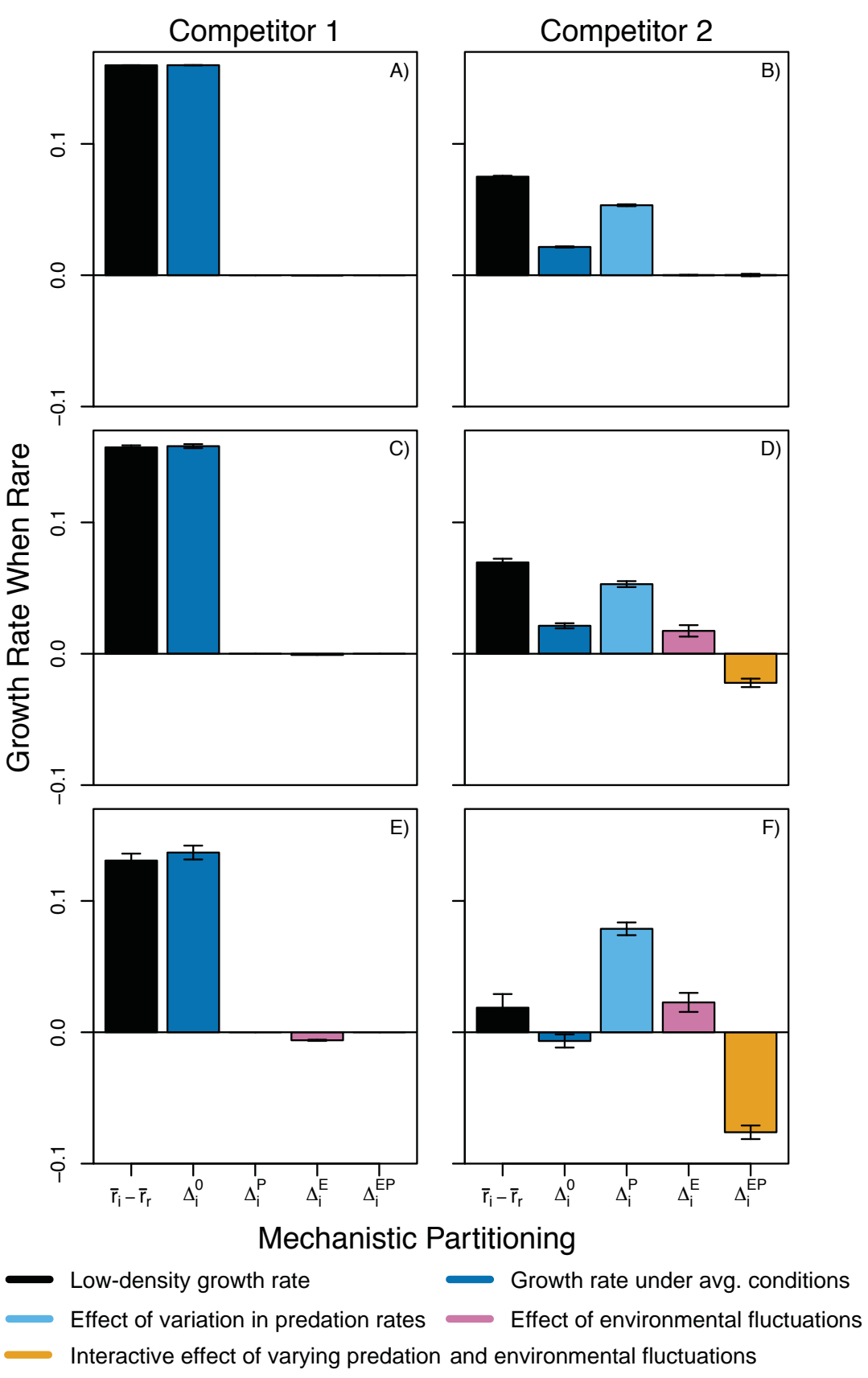

Figure S2.4: Increasing the strength of environmental variation $(\sigma)$ on coexistence mechanisms. The top row (A, B) shows low environmental variation $(\sigma=0.1)$, while the middle row $(\mathrm{C}, \mathrm{D})$ shows medium environmental variation $(\sigma=0.4)$, and the last row $(\mathrm{E}, \mathrm{F})$ shows high environmental variation $(\sigma=0.7)$. Each subpanel shows results from 500 runs, where error bars denote standard deviation. 
Quantifying the relative importance of variation in predation and the environment for species coexistence

\title{
Expanding the Diamond Model for Additional Complexity: Supplement 3
}

\author{
Lauren G. Shoemaker, Allison K. Barner, Leonora S. Bittleston, and Ashley I. Teufel
}

\section{Three Consumers, One Predator}

Expanding on the diamond model to include a third competitor yields the following set of equations:

$$
\begin{gathered}
\frac{d P}{d t}=-M_{p} P+\frac{J_{p} P\left[\Omega_{P C_{1}} C_{1}+\Omega_{P C_{2}} C_{2}+\left(1-\left(\Omega_{P C_{1}}+\Omega_{P C_{2}}\right)\right) C_{3}\right]}{\Omega_{P C_{1}} C_{1}+\Omega_{P C_{2}} C_{2}+\left(1-\left(\Omega_{P C_{1}}+\Omega_{P C_{2}}\right)\right) C_{3}+C_{0}} \\
\frac{d C_{1}}{d t}=-M_{C_{1}} C_{1}+\frac{\Omega_{C_{1} R} J_{C_{1}} C_{1} R}{R+R_{0_{1}}}-\frac{\Omega_{P C_{1}} J_{p} P C_{1}}{\Omega_{P C_{1}} C_{1}+\Omega_{P C_{2}} C_{2}+\left(1-\left(\Omega_{P C_{1}}+\Omega_{P C_{2}}\right)\right) C_{3}+C_{0}} \\
\frac{d C_{2}}{d t}=-M_{C_{2}} C_{2}+\frac{\Omega_{C_{2} R} J_{C_{2}} C_{2} R}{R+R_{0_{2}}}-\frac{\Omega_{P C_{2}} J_{p} P C_{2}}{\Omega_{P C_{1}} C_{1}+\Omega_{P C_{2}} C_{2}+\left(1-\left(\Omega_{P C_{1}}+\Omega_{P C_{2}}\right)\right) C_{3}+C_{0}} \\
\frac{d C_{3}}{d t}=-M_{C_{3}} C_{3}+\frac{\Omega_{C_{3} R} J_{C_{3}} C_{3} R}{R+R_{0_{3}}}-\frac{\left(1-\left(\Omega_{P C_{1}}+\Omega_{P C_{2}}\right)\right) J_{p} P C_{3}}{\Omega_{P C_{1}} C_{1}+\Omega_{P C_{2}} C_{2}+\left(1-\left(\Omega_{P C_{1}}+\Omega_{P C_{2}}\right)\right) C_{3}+C_{0}} \\
\frac{d R}{d t}=r R(1-R / K)-\frac{\Omega_{C_{1} R} J_{C_{1}} C_{1} R}{R+R_{0_{1}}}-\frac{\Omega_{C_{2} R} J_{C_{2}} C_{2} R}{R+R_{0_{2}}}-\frac{\Omega_{C_{3} R} J_{C_{3}} C_{3} R}{R+R_{0_{3}}} \\
\text { where }\left(\Omega_{P C_{1}}+\Omega_{P C_{2}}\right)<=1
\end{gathered}
$$

where parameter values are given in the main text (Table 1). We estimated parameters of this system twice, allowing us to compare coexistence under the same food web structure, but with different interaction strengths.

To confirm that the estimated parameters result in stable dynamics we examine the dynamics of both of these systems without variance (Fig. S3.1, S3.2) and with variance (Fig. S3.3, S3.4). 

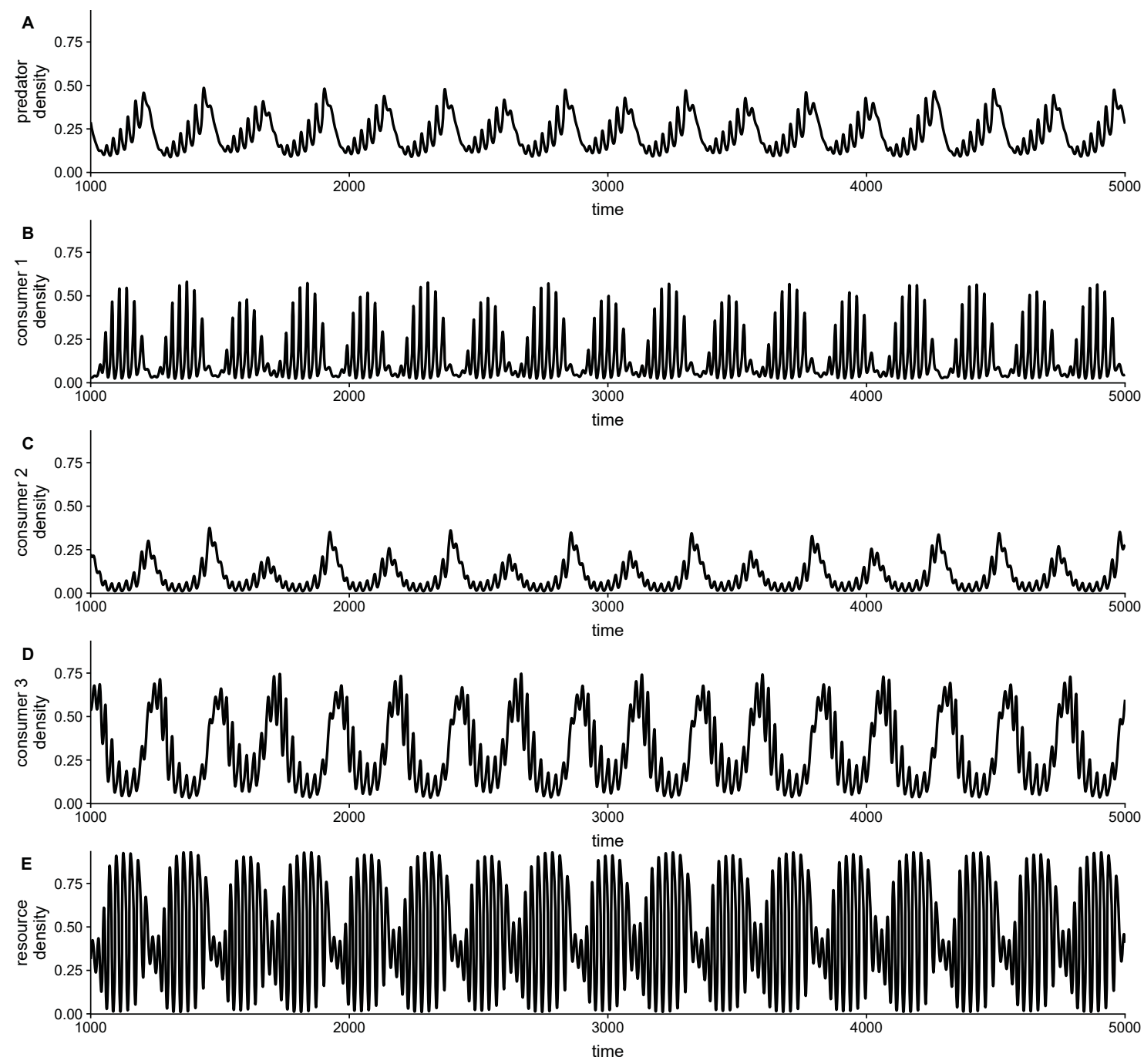

Figure S3.1: Dynamics of the 3 competitor system, from the first set of parameters in the main text (Table 1), as denoted in bold. The figure shows dynamics without environmental perturbations altering mortality rates. 

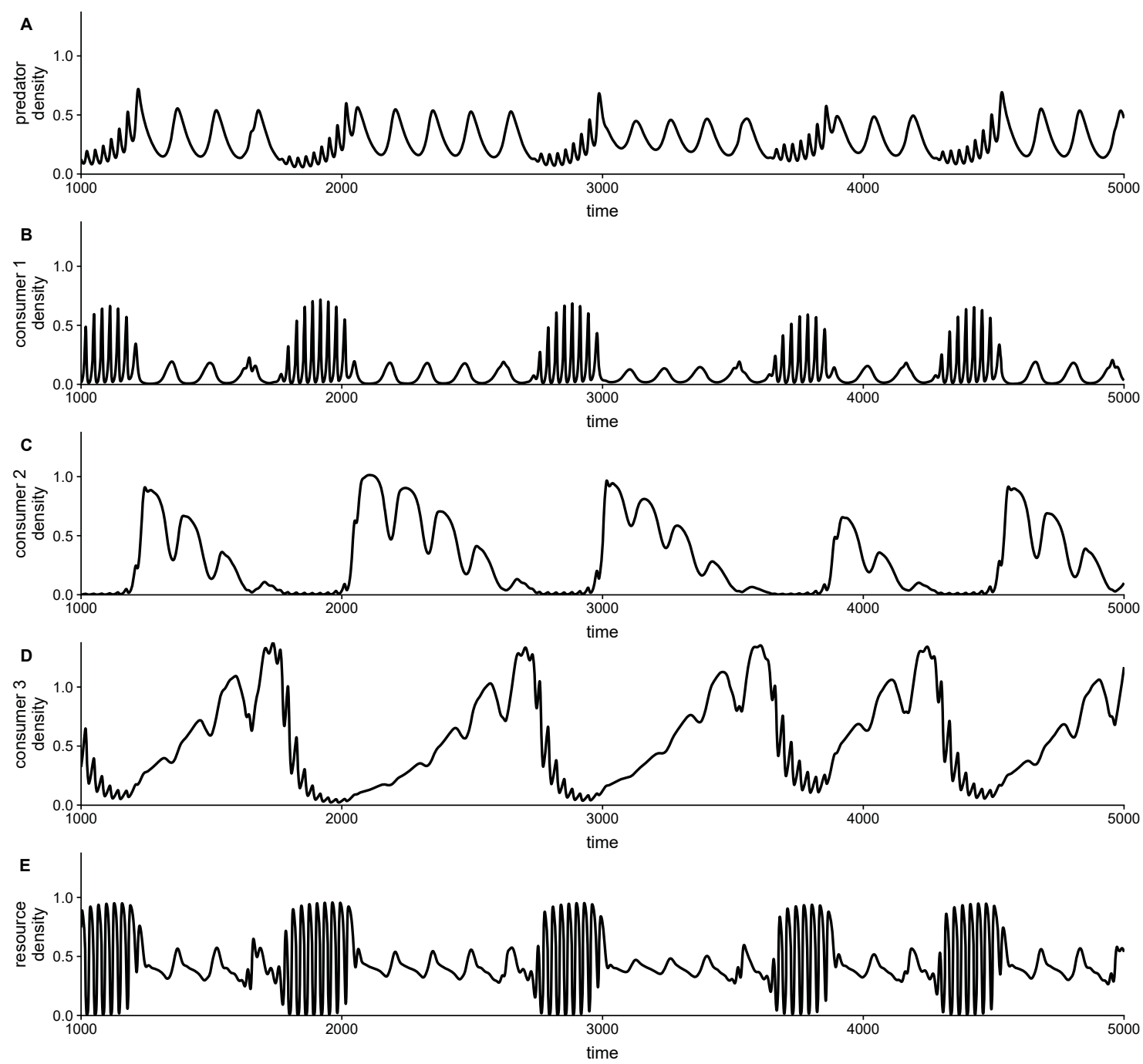

Figure S3.2: Dynamics of a 3 competitor system. These values of the parameters are the second set of parameters given in Table 1. The figure shows dynamics without environmental perturbations altering mortality rates. 

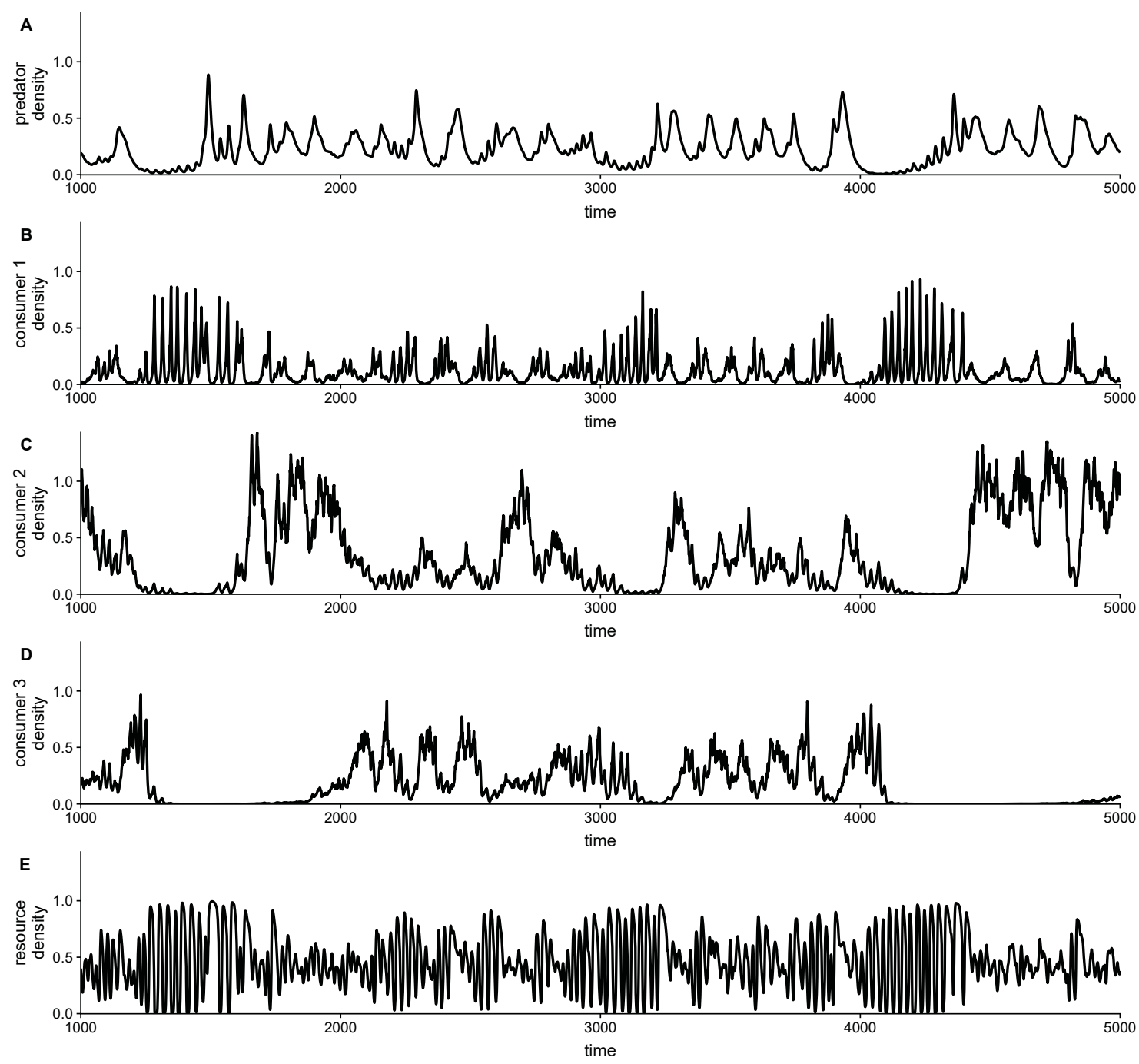

Figure S3.3: Example dynamics of a 3 competitor system (Fig. S3.1) when competitor mortality is impacted by environmental fluctuations. 

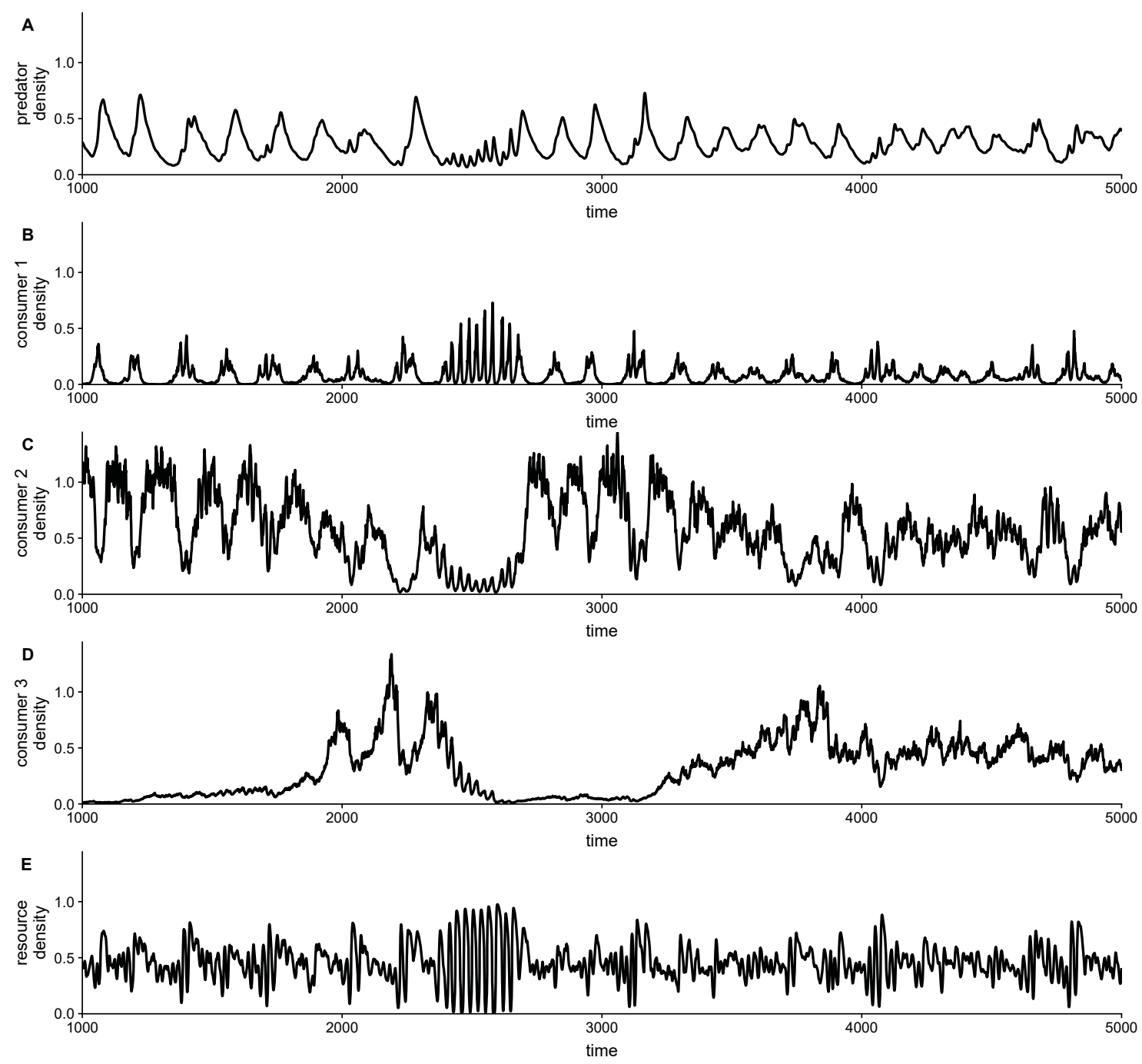

Figure S3.4: Example dynamics of a 3 competitor system (Fig. S3.2) when competitor mortality is impacted by environmental fluctuations. 


\section{Three Consumers, Two Predators}

Expanding the model further to include a second predator yields:

$$
\begin{aligned}
& \frac{d P_{1}}{d t}=-M_{p_{1}} P_{1}+\frac{J_{p_{1}} P_{1}\left[\Omega_{P_{1} C_{1}} C_{1}+\Omega_{P_{1} C_{2}} C_{2}+\left(1-\left(\Omega_{P_{1} C_{1}}+\Omega_{P_{1} C_{2}}\right) C_{3}\right]\right.}{\tau_{1}} \\
& \frac{d P_{2}}{d t}=-M_{p_{2}} P_{2}+\frac{J_{p_{2}} P_{2}\left[\Omega_{P_{2} C_{1}} C_{1}+\Omega_{P_{2} C_{2}} C_{2}+\left(1-\left(\Omega_{P_{2} C_{1}}+\Omega_{P_{2} C_{2}}\right)\right) C_{3}\right]}{\tau_{2}} \\
& \frac{d C_{1}}{d t}=-M_{C_{1}} C_{1}+\frac{\Omega_{C_{1} R} J_{C_{1}} C_{1} R}{R+R_{0_{1}}}-\frac{\Omega_{P_{1} C_{1}} J_{p 1} P_{1} C_{1}}{\tau_{1}}-\frac{\Omega_{P_{2} C_{1}} J_{p 2} P_{2} C_{1}}{\tau_{2}} \\
& \frac{d C_{2}}{d t}=-M_{C_{2}} C_{2}+\frac{\Omega_{C_{2} R} J_{C_{2}} C_{2} R}{R+R_{0_{2}}}-\frac{\Omega_{P_{1} C_{2}} J_{p 1} P_{1} C_{2}}{\tau_{1}}-\frac{\Omega_{P_{2} C_{2}} J_{p 2} P_{2} C_{2}}{\tau_{2}} \\
& \frac{d C_{3}}{d t}=-M_{C_{3}} C_{3}+\frac{\Omega_{C_{3} R} J_{C_{3}} C_{3} R}{R+R_{0_{3}}}- \\
& \frac{\left(1-\left(\Omega_{P_{1} C_{1}}+\Omega_{P_{1} C_{2}}\right)\right) J_{p_{1}} P_{1} C_{3}}{\tau_{1}}-\frac{\left(1-\left(\Omega_{P_{2} C_{1}}+\Omega_{P_{2} C_{2}}\right)\right) J_{p_{2}} P_{2} C_{3}}{\tau_{2}} \\
& \frac{d R}{d t}=r R(1-R / K)-\frac{\Omega_{C_{1} R} J_{C_{1}} C_{1} R}{R+R_{0_{1}}}-\frac{\Omega_{C_{2} R} J_{C_{2}} C_{2} R}{R+R_{0_{2}}}-\frac{\Omega_{C_{3} R} J_{C_{3}} C_{3} R}{R+R_{0_{3}}}
\end{aligned}
$$

where

$\tau_{1}=\Omega_{P_{1} C_{1}} C_{1}+\Omega_{P_{1} C_{2}} C_{2}+\left(1-\left(\Omega_{P_{1} C_{1}}+\Omega_{P_{1} C_{2}}\right)\right) C_{3}+C_{0_{P_{1}}}$

$\tau_{2}=\Omega_{P_{2} C_{1}} C_{1}+\Omega_{P_{2} C_{2}} C_{2}+\left(1-\left(\Omega_{P_{2} C_{1}}+\Omega_{P_{2} C_{2}}\right)\right) C_{3}+C_{0_{P_{2}}}$.

We again confirm that estimated parameters result in stable dynamics in the absence of variability in mortality rates before examining coexistence with both environmental and predator fluctuations. 

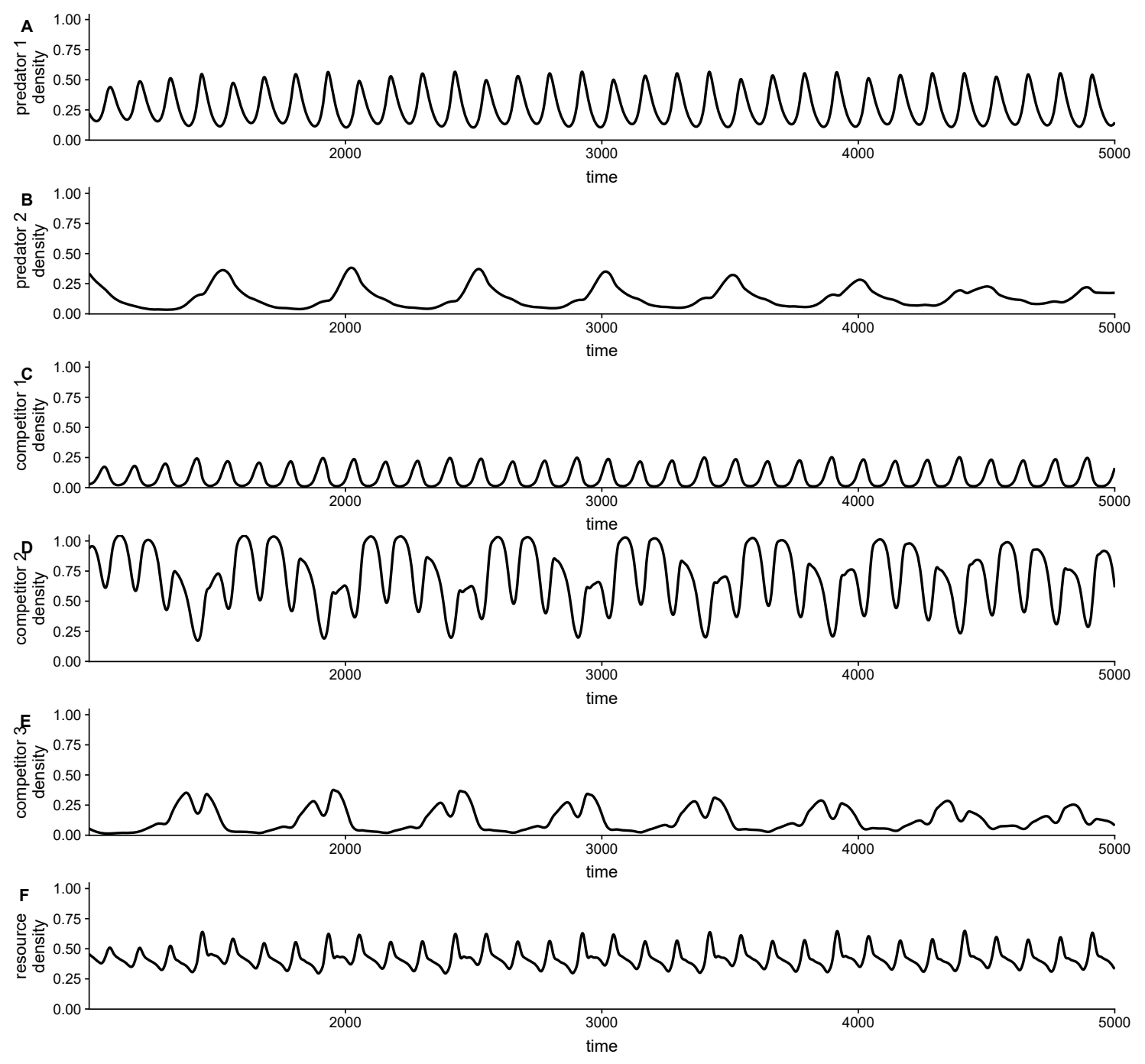

Figure S3.5: Dynamics of the 3 competitor and 2 predator system in the absence of variation in mortality rates. Parameters are given in Table 1 of the main text. 

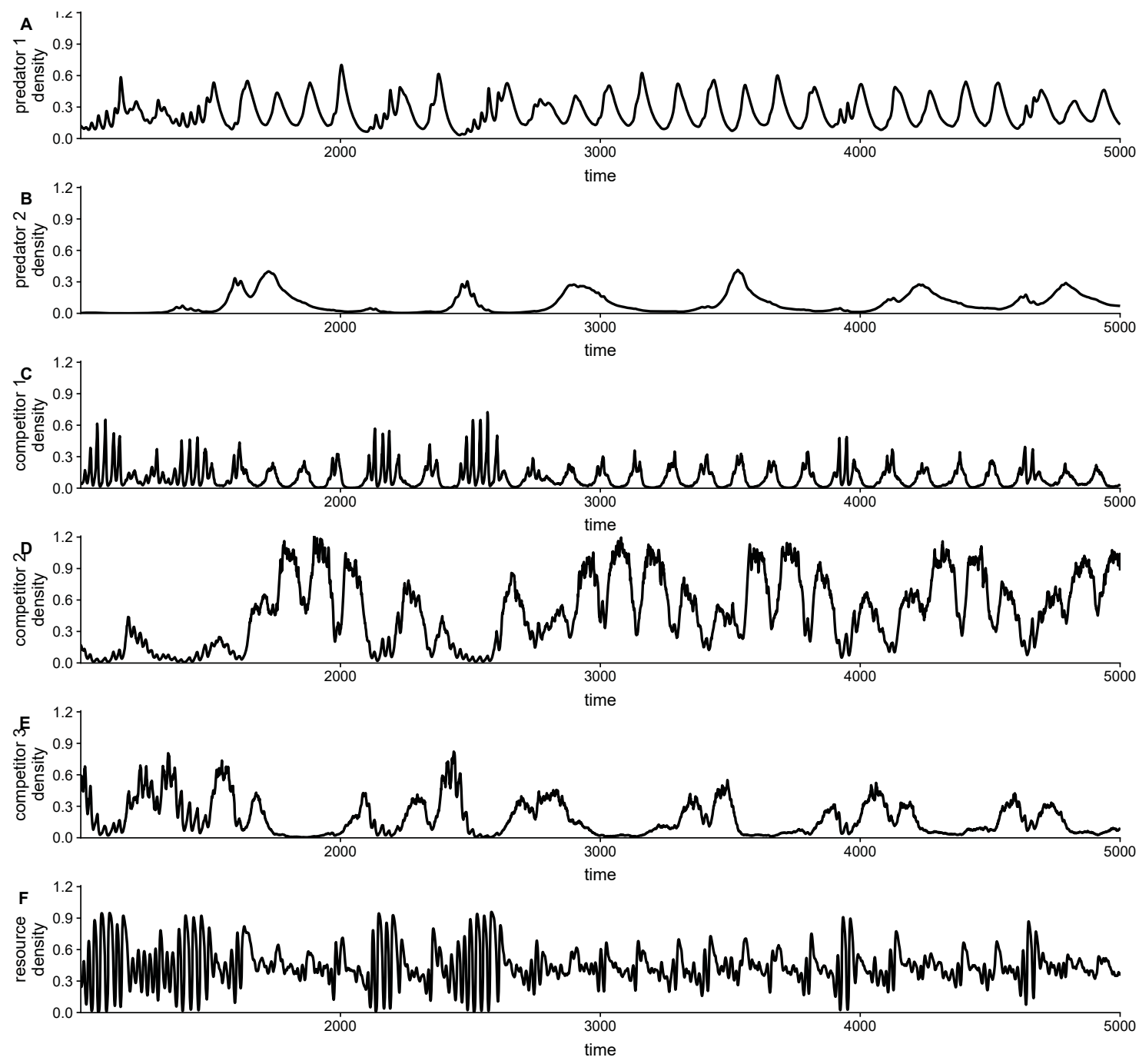

Figure S3.6: Example dynamics of the 3 competitor and 2 predator system with environmental variation. Parameters are given in Table 1 of the main text. 


\section{Coexistence Comparisons}

System

A)<smiles>[R]1[C]P[Te]1</smiles>

System

D)<smiles>[R]C1CCCCC1</smiles>

System

H)

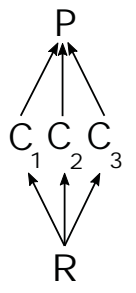

Competitor 1

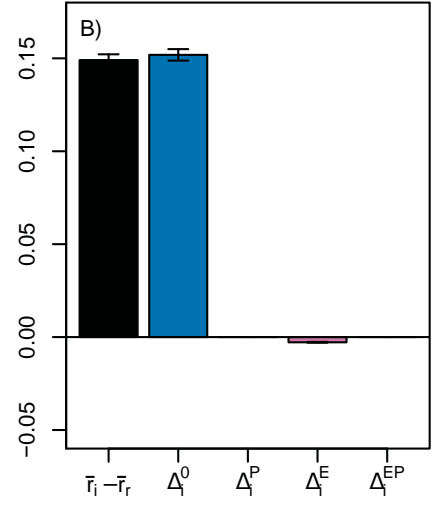

Competitor 2

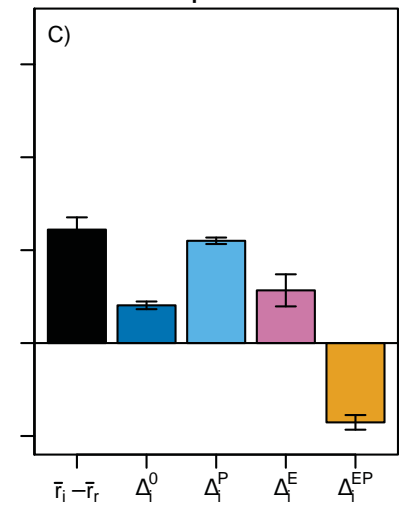

Competitor 1

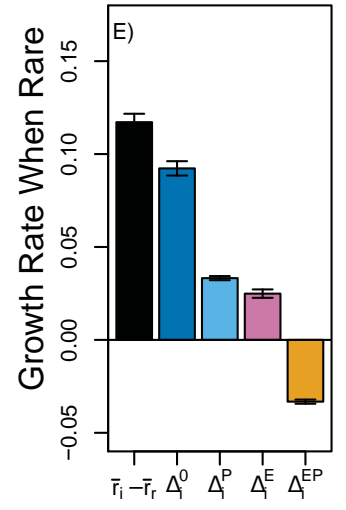

Competitor 2

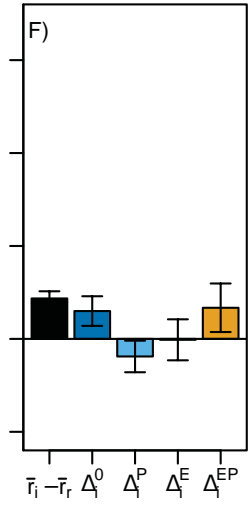

Competitor 3

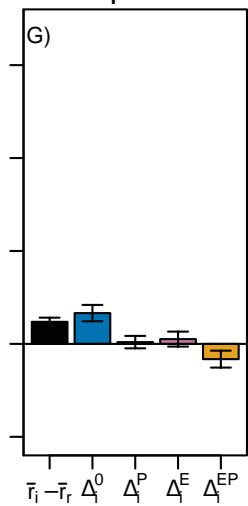

Competitor 3

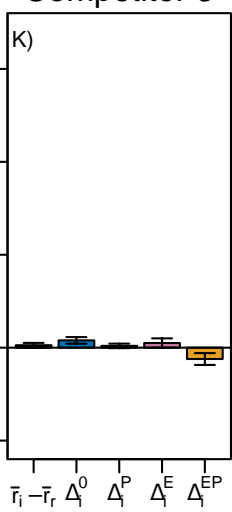

Mechanistic Partitioning

Competitor

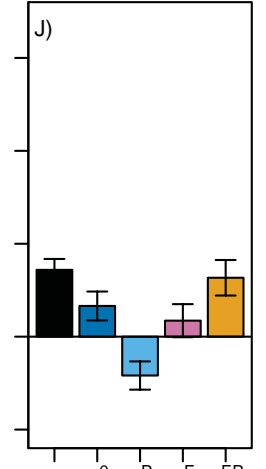

\section{ing}
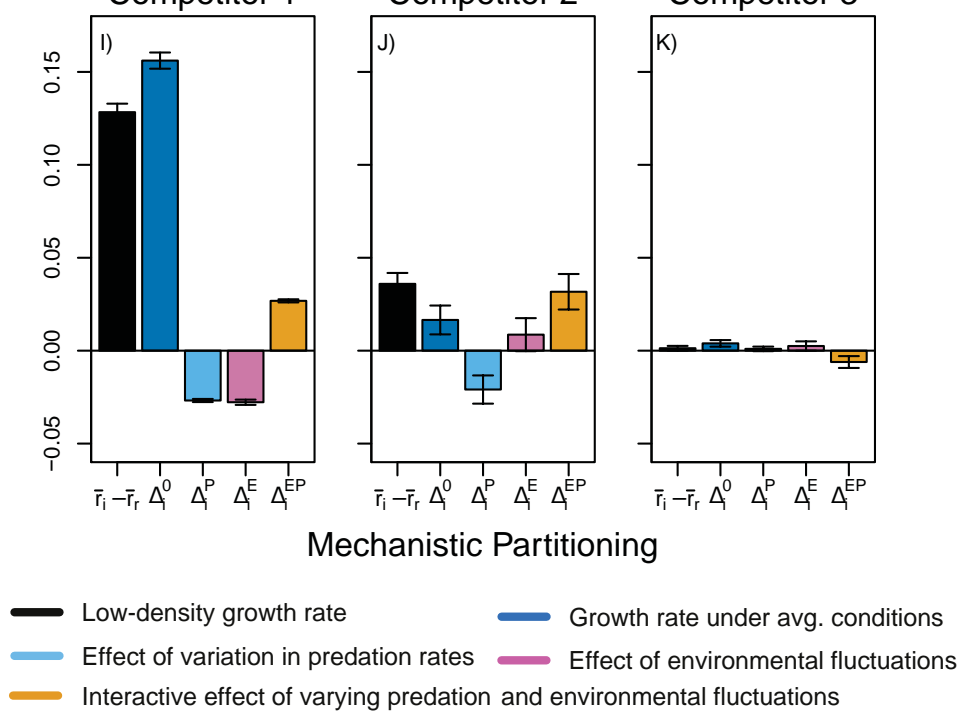

Figure S3.7: Decomposition of the 3 competitor model under two different characterizations. (A-C) Replica of results from the diamond model, shown here to facilitate comparison of three competitor models. (D-G) Three competitor model under the parameter set from replicate 1 , as shown in bold in Table 1 of the main text. Data shown here is a replica of data shown in main text to facilitate comparison. (H-K) Three competitor model under the parameter set from replicate 2 given in Table 1 in the main text. 


\title{
Quantifying the relative importance of variation in predation and the environment for species coexistence
}

\author{
Rocky Intertidal Food Web Case Study: Supplement 4
}

\author{
Lauren G. Shoemaker, Allison K. Barner, Leonora S. Bittleston, and Ashley I. Teufel
}

\section{Model}

Our model for rocky intertidal food web dynamics closely builds on the model proposed by Forde \& Doak (2004). This model is an extension of foundational work by Iwasa \& Roughgarden (1986) and Connolly \& Roughgarden (1999). We generally followed the model proposed by Forde \& Doak (2004), but made several small changes to facilitate model implementation. Equations S4.3, S4.7, S4.8, and S4.10 were modified from Forde \& Doak (2004), described below.

Free space at each time step is a function of the total available space $\left(T_{t}\right)$ and the area taken up by the three spacelimited competitors (B. glandula, $C$. dalli, and limpets). The total area for each of the three competitor species is proportional to their population size (e.g., $B_{t}$ ) and the average size of an adult individual (e.g., $A_{b}$, Table S4.1). Free space is limited to between 0 and $1\left(\mathrm{~m}^{2}\right.$, see Section 2 for more on parameters and units). Free space at time $t$ follows:

$$
F_{t}=T_{t}-\left(B_{t} A_{b}+C_{t} A_{c}+L_{t} A_{l}\right)
$$

Barnacles and limpets have pelagic larvae, so recruitment at a given time step (i.e. month) is not related to local abundance and is instead a mass-action process (Connolly and Roughgarden, 1999; Wieters et al., 2008). For each of the these three competitor species, recruitment was thus modeled as a function of available space for the species at the last time point, representing the potential (or maximum) recruitment at a given time step. The number of larvae $\left(m_{x}\right)$ in the water column is randomly drawn from a lognormal distribution at each time step (Table 1 in Forde \& Doak 2004), but the total recruitment from this larval pool can never be larger than the amount of available space. The size of the larval pool at time $t$ for species $X \subset\{B, C, L\}$ :

$$
M_{x, t}=\frac{F_{t-1}}{A_{x}}\left[1-e^{-a_{x} m_{x} / F_{t-1}}\right]
$$

Realized barnacle and limpet recruitment was based on Equation 1 in Iwasa and Roughgarden (1986), where settlement of planktonic larvae into the local system depends on the amount of free space at the last time step, the number of larvae of the species in the larval pool (the "potential" recruitment), and the rate of larval settlement of the species (Table S4.1). Actual (realized) recruitment at time $t$ for species $X \subset\{B, C, L\}$ :

$$
R_{x, t}=d_{x} M_{t} F_{t-1}
$$

For the two barnacle species, population size at a given time point is a function of adult survivorship from the previous time step, density-independent survival of recruits from the larval pool, and the loss of adults to sea star and whelk predation. Following the assumption of Forde \& Doak (2004), recruits transition into the adult population after one month. Population size for barnacle species $Y \subset\{B, C\}$ at time $t$ :

$$
Y_{t}=Y_{t-1} S_{y}+s_{y} R_{y, t}-p_{w h e l k} W_{t-1} Y_{t-1} S_{y}-p_{\text {seastar }} P_{t-1} Y_{t-1} S_{y}
$$

The Forde \& Doak limpet population model, unlike barnacles, does not include explicit mortality due to predation. Instead, predation on the limpet population is implicitly modeled as density dependence. Thus, limpet population size is a function of adult survival from the previous time step, and density-dependent survival of recruits from the larval pool. Density-dependence, following Forde \& Doak (2004), is given by a parameter $\delta$ (Table S4.1). According to Forde \& Doak, delta was used to model the density-dependence that would occur if limpet predators were included in the model, but no justification for the parameter value was given. Future work could explicitly include predator dynamics, as in NE Pacific intertidal systems, limpets are eaten by surfperch (Mercurio et al., 1985), birds (Marsh, 1986), sea stars (Phillips and Castori, 1982), and crabs (Lowell, 1986). Similar to barnacles, limpet larval supply is modeled using random draws from a lognormal distribution (Table 1 in Forde \& Doak 2004). Population size for limpets at time $t$ :

$$
L_{t}=S_{l} L_{t-1}+s_{l} R_{l, t} e^{\delta L_{t-1}}
$$


Whelks lay egg masses once per year, unlike all other species in the model with planktonic larvae. As such, recruitment was calculated once a year (June), and modeled as follows from Forde \& Doak (2004). The potential number of new whelk recruits in June was a function of the barnacle prey consumed in the previous three months. $C_{\text {average }}$ and $B_{\text {average }}$ are the average number of barnacle prey available in April to June, $p_{w}$ is whelk predation rate, and $\gamma$ is the predator conversion rate. Thus, potential whelk recruitment at time $t$ :

$$
M_{w, t}=\left(C_{\text {average }}+B_{\text {average }}\right) 3 p_{w h e l k} \gamma S_{w, t}\left(C_{t-1}+B_{t-1}\right)
$$

In the original Forde \& Doak model (2004), a step function was used to implement density-dependence in whelk recruitment (e.g., if $R_{w}>90$, then set $R_{w}=90$ ). Here, we instead used a discrete logistic population equation, with a carrying capacity $(K)$ of 90 . This required an additional assumption of the population growth rate $(r$, Table S4.1). Actual (realized) whelk recruitment at time $t$ :

$$
R_{w, t}=M_{w, t} \frac{M_{w, t}}{K_{w}} e^{r_{w}\left(1-\frac{M_{w, t}}{K_{w}}\right)}
$$

To model whelk adult population size, we simplified the equation from Forde \& Doak 2004, so that the whelk population size is modeled similarly to that of the other predator in the system (sea stars; Connolly and Roughgarden (1999)). Population size is simply a function of adults that survived from the previous month (with constant per capita mortality) and new whelk recruits (if June). Population size for whelks at time $t$ :

$$
W_{t}=W_{t-1} * S_{w}+R_{w, t}
$$

The larval pool for predator sea stars (Pisaster ochraceus), like barnacles and limpets, was modeled as random draws from a lognormal distribution (Table 1 in Forde \& Doak 2004). However, sea stars are assumed to not compete for space with barnacles and limpets in this model. Thus, sea star recruitment is simply a function of the larval pool in the water column at a given point in time, and is not related to the available free space. Sea star recruitment at time $t$ :

$$
\ln \left(R_{p, t}\right) \sim \mathcal{N}\left(\mu_{p}, \sigma_{p}^{2}\right)
$$

Similar to the modified whelk recruitment equation, the population model for sea star abundance was modified from Forde \& Doak 2004 to explicitly incorporate density dependence. The step function of Forde \& Doak (if $P_{t}<6, P_{t}$ $=P_{t}$, else $P_{t}=6$ ) was updated to saturate at a carrying capacity of 6 , with population size a function of adult survival at the last time point and recruitment into the system. Note that unlike whelk population size, sea star populations are not a function of local prey abundance (Wieters et al., 2008) because adult sea star abundance is instead related to the size of the regional larval pool (Connolly and Roughgarden, 1999). Sea star population size at time $t$ :

$$
P_{t}=\rho_{t} \frac{\rho_{t}}{K_{p}} e^{r_{p}\left(1-\frac{\rho_{t}}{K_{p}}\right)}
$$

Where, $\rho_{t}=\left(S_{p} P_{t-1}\right)+\left(s_{p} R_{p}\right)$.

\section{Parameterization}

When possible, we used the parameters from Forde \& Doak (2004) Tables 1 and 2; all parameters came from: Burrows and Hughes (1991); Forde (2002); Forde and Doak (2004); Frank (1965); Menge et al. (1994), and Palmer (1990) (Table S4.1). We modified the realized recruitment equation for barnacles and limpets (equation S4.3), which included a new per capita settlement parameter, $d$. Following Connolly \& Roughgarden (1999), the settlement coefficient was the same for all three species (see also Gilman (2006) for independent derivation of settlement rate for limpets). Further, the original Forde \& Doak model did not include survival rates for sea star recruits. We calculated recruit survival from Menge (1975), given two pieces of information: the average annual survival of spawned gametes to postmaturity longevity is $1.46 \times 10^{-9} / \mathrm{m}^{2} /$ year and the annual mortality of gametes is 0.999 Menge (1975). Whelk and sea star population models were rewritten from a step function in Forde \& Doak (2004) to a density-dependent logistic form. To do so, we simply assumed the population growth rate of sea stars was 1, while the whelk population growth rate was much lower. The lower whelk population growth rate was set lower $(0.3)$ after preliminary runs of the model found that a growth rate of 1 resulted in very strong density-dependence that held the whelk population size at fewer than 1 -well below the initial population size of 93.

We used the same barnacle adult and recruit survival rates as Forde \& Doak (2004). Future work could explore model dynamics if Balanus survives at a higher rate than Chthamalus, as has been empirically shown in Connell (1961). 
Similarly, we continue to use the model form of Forde \& Doak such that neither predator has a prey preference, although past experiments suggest that Balanus is predated at a higher rate than Chthamalus (Connell, 1961; Navarrete et al., 2000). Instead, we increased the per-capita rate of predation to mimic the effect of strong predation on barnacles. Finally, the initial population size was set to be the same for both barnacle species, and was set to the lower initial population size given for Balanus in Forde \& Doak (2004). Thus, the only difference in the model for the two barnacle species is adult size (Balanus $>$ Chthamalus) and larval supply rates under "high" supply scenarios (also Balanus $>$ Chthamalus).

Larval supply rates for barnacles, Pisaster ochraceous, and limpets were identical to those in Table 1 in Forde \& Doak (2004). For each species, there were three mean values (low, medium, high) and for each mean value, there were three variance values (low, medium, high). For all simulations, we used the "low" variance option that was associated with each of the "low" and "high" mean larval supply values. 


\begin{tabular}{|c|c|c|c|}
\hline Parameter & Description & Value & Source \\
\hline \multicolumn{4}{|c|}{ Balanus glandula (B) } \\
\hline$B_{0}$ & Initial population size & $4100 \mathrm{~m}^{-2}$ & Forde (2002) \\
\hline$S_{B}$ & Adult survival rate & $0.7 \mathrm{mo}^{-1}$ & $\begin{array}{l}\text { Connolly \& Roughgarden } \\
\text { (1999) }\end{array}$ \\
\hline$S_{b}$ & Recruit survival rate & $0.7 \mathrm{mo}^{-1}$ & $\begin{array}{l}\text { Connolly \& Roughgarden } \\
\text { (1999) }\end{array}$ \\
\hline$A_{B}$ & Average adult size & $0.98 \mathrm{~cm}^{-2}$ & Forde (2002) \\
\hline$A_{b}$ & Average recruit size & $0.03 \mathrm{~cm}^{-2}$ & Forde (2002) \\
\hline$d_{b}$ & Larval settlement coefficient* & $1.44 \mathrm{mo}^{-1}$ & $\begin{array}{l}\text { Connolly \& Roughgarden } \\
\text { (1999) }\end{array}$ \\
\hline \multicolumn{4}{|c|}{ Chthamalus fissus/dalli (C) } \\
\hline$C_{0}$ & Initial population size & $4100 \mathrm{~m}^{-2}$ & Forde (2002) \\
\hline$S_{C}$ & Adult survival rate & $0.7 \mathrm{mo}^{-1}$ & $\begin{array}{l}\text { Connolly \& Roughgarden } \\
\text { (1999) }\end{array}$ \\
\hline$S_{c}$ & Recruit survival rate & $0.7 \mathrm{mo}^{-1}$ & $\begin{array}{l}\text { Connolly \& Roughgarden } \\
\text { (1999) }\end{array}$ \\
\hline$A_{C}$ & Average adult size & $0.32 \mathrm{~cm}^{-2}$ & Forde (2002) \\
\hline$A_{c}$ & Average recruit size & $0.03 \mathrm{~cm}^{-2}$ & Forde (2002) \\
\hline$d_{c}$ & Larval settlement coefficient* & $1.44 \mathrm{mo}^{-1}$ & $\begin{array}{l}\text { Connolly \& Roughgarden } \\
\text { (1999) }\end{array}$ \\
\hline \multicolumn{4}{|l|}{ Limpets (L) } \\
\hline$L_{0}$ & Initial population size & $239 \mathrm{~m}^{-2}$ & Forde (2002) \\
\hline$S_{L}$ & Adult survival rate & $0.97 \mathrm{mo}^{-1}$ & Frank (1965) \\
\hline$S_{l}$ & Recruit survival rate & $0.88 \mathrm{mo}^{-1}$ & Frank (1965) \\
\hline$A_{L}$ & Average adult size & $0.8 \mathrm{~cm}^{-2}$ & Forde (2002) \\
\hline$A_{l}$ & Average recruit size & $0.03 \mathrm{~cm}^{-2}$ & Forde (2002) \\
\hline$d_{l}$ & Larval settlement coefficient* & $1.44 \mathrm{mo}^{-1}$ & $\begin{array}{l}\text { Connolly \& Roughgarden } \\
\text { (1999) }\end{array}$ \\
\hline$\delta$ & Density-dependent paramater & -0.02 & Forde \& Doak (2004) \\
\hline \multicolumn{4}{|l|}{ Whelks (W) } \\
\hline$W_{0}$ & Initial population size & $93 \mathrm{~m}^{-2}$ & Forde (2002) \\
\hline$r_{w}$ & $\begin{array}{l}\text { Population growth rate of whelk } \\
\text { recruits* }\end{array}$ & 0.3 & \\
\hline$S_{W}$ & Adult survival rate & $0.94 \mathrm{mo}^{-1}$ & Burrows \& Hughes (1991) \\
\hline$S_{w}$ & Recruit survival rate* & $0.88 \mathrm{mo}^{-1}$ & \\
\hline$K_{W}$ & Carrying capacity* & 90 & Forde \& Doak (2004) \\
\hline$p_{W, B}$ & $\begin{array}{l}\text { Per capita predation rate on } \\
\text { Balanus* }\end{array}$ & 0.02 & Forde \& Doak (2004) \\
\hline$p_{W, C}$ & $\begin{array}{l}\text { Per capita predation rate on } \\
\text { Chthamalus* }\end{array}$ & 0.02 & Forde \& Doak (2004) \\
\hline $\begin{array}{c}Y_{W} \\
\text { Pisaster ochraceu }\end{array}$ & Conversion rate & 0.001 & Forde \& Doak (2004) \\
\hline$P_{0}$ & Initial population size & $1 \mathrm{~m}^{-2}$ & Menge et al. (1994) \\
\hline$r_{p}$ & $\begin{array}{l}\text { Population growth rate of adult } \\
\text { sea stars* }\end{array}$ & 1.0 & \\
\hline$S_{P}$ & Adult survival rate & $0.992 \mathrm{mo}^{-1}$ & $\begin{array}{l}\text { Connolly \& Roughgarden } \\
\text { (1999) }\end{array}$ \\
\hline$S_{w}$ & Recruit survival rate* & $0.001 \mathrm{mo}^{-1}$ & Menge (1975) \\
\hline$K_{W}$ & Carrying capacity* & 6 & Forde \& Doak (2004) \\
\hline$p_{W, B}$ & $\begin{array}{l}\text { Per capita predation rate on } \\
\text { Balanus* }\end{array}$ & 0.02 & Forde \& Doak (2004) \\
\hline$p_{W, C}$ & $\begin{array}{l}\text { Per capita predation rate on } \\
\text { Chthamalus* }\end{array}$ & 0.02 & Forde \& Doak (2004) \\
\hline
\end{tabular}

Table S4.1: Parameters used in the model. Descriptions marked with an asterisk indicate parameters that were not included in or modified from the original Forde \& Doak (2004) model. 
This is an author-produced, peer-reviewed version of this article. The final, definitive version of this document can be found online at Ecology Letters, published by John Wiley \& Sons Ltd/CNRS. Copyright restrictions may apply. doi: 10.1111/ele.13482
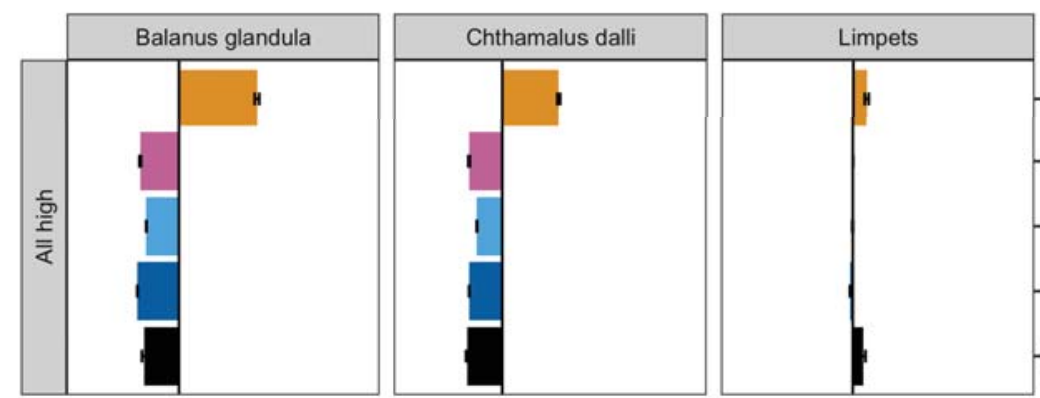

ธั)
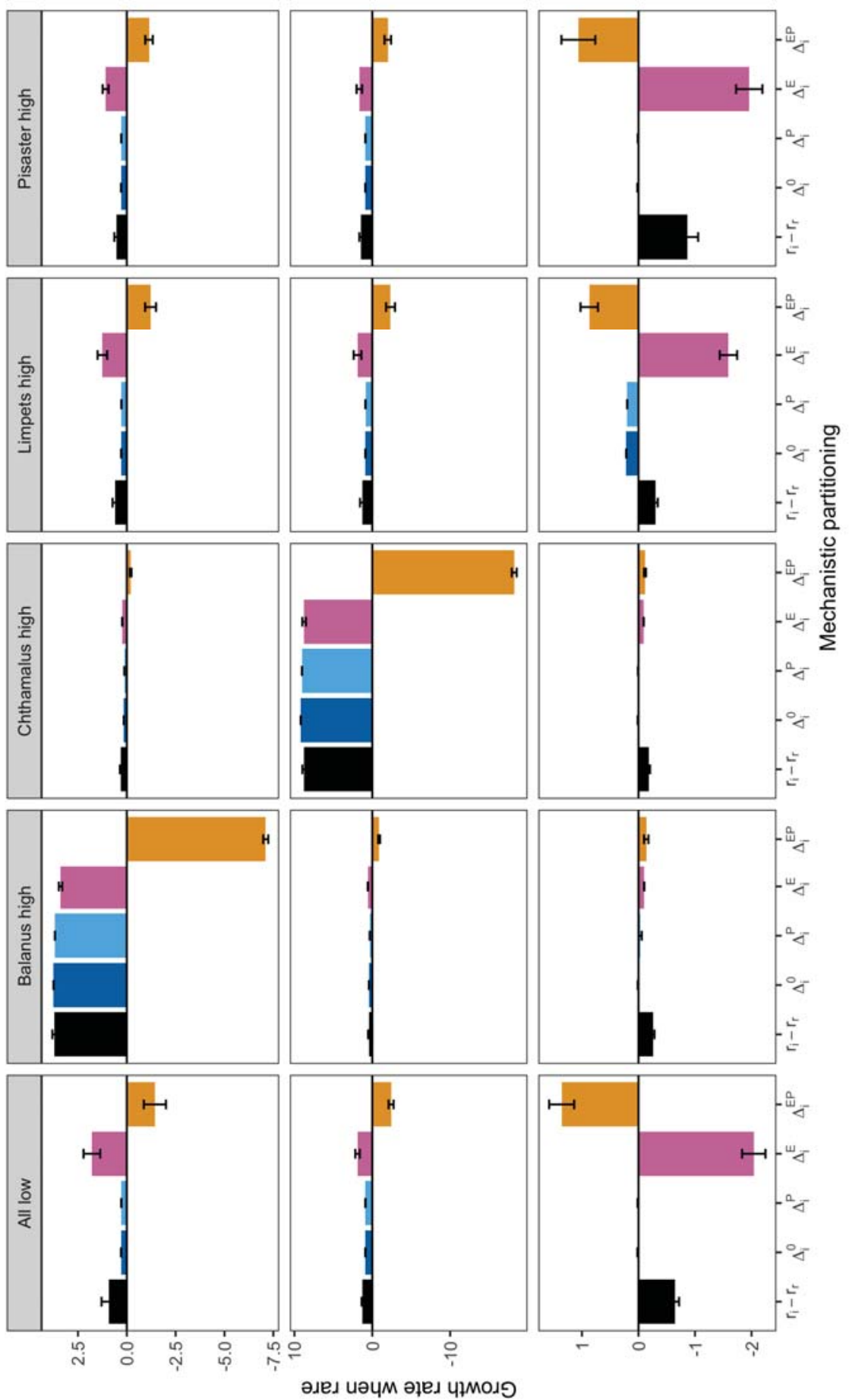

牙

ב

突.

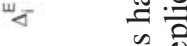

$4 \quad-\frac{\infty}{4}$

की

필

(马)

클

ํㅡㄹ

츙

도을

خे

ڤ્

สำ

크

.$\Xi$

$\frac{n}{2}$

टे

츤 궁

o

字

क्षे

ฮ

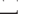

胥

궁

클

次

츨

ब

주

을

.0.

ธี

先

ปี

8

ये के

등

胥

.

这密

$\therefore$ 궁

구응

흘

인을 

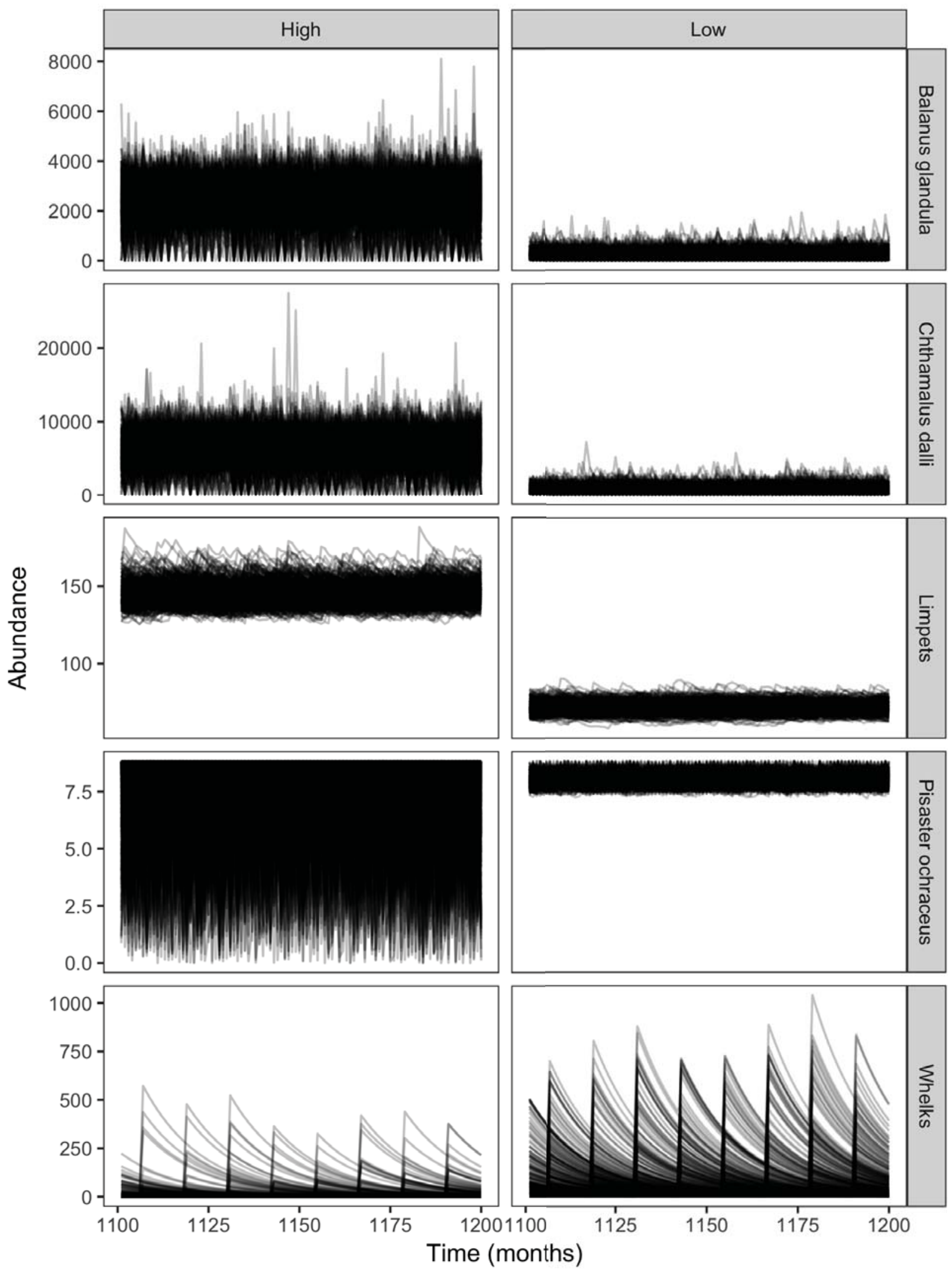

Figure S4.2: Last 100 time steps (months) for 500 runs of the intertidal model, under "low" and "high" larval supply. Here, all species were started at their initial population size given in Table S4.1 and then run with all species having either "low" or "high" larval supply, as given in Forde \& Doak Table 1. 


\section{References}

Burrows, M. T. and Hughes, R. N. (1991). Optimal foraging decisions by dogwhelks, Nucella lapillus (L.): influences of mortality risk and rate-constrained digestion. Functional Ecology, 5:461-475.

Connell, J. H. (1961). The influence of interspecific competition and other factors on the distribution of the barnacle Chthamalus stellatus. Ecology, 42:710-723.

Connolly, S. R. and Roughgarden, J. (1999). Theory of marine communities: competition, predation, and recruitmentdependent interaction strength. Ecological Monographs, 69:277-296.

Forde, S. (2002). The effects of reruitment variability on population and community dynamics. Ph.D. thesis, University of California, Santa Cruz.

Forde, S. E. and Doak, D. F. (2004). Multitrophic interactions mediate recruitment variability in a rocky intertidal community. Marine Ecology Progress Series, 275:33-45.

Frank, P. W. (1965). The biodemography of an intertidal snail population. Ecology, 46:831-844.

Gilman, S. E. (2006). The northern geographic range limit of the intertidal limpet Collisella scabra: a test of performance, recruitment, and temperature hypotheses. Ecography, 5:709-720.

Iwasa, Y. and Roughgarden, J. (1986). Interspecific competition among metapopulations with space-limited subpopulations. Theoretical Population Biology, 30:194-214.

Lowell, R. B. (1986). Crab predation on limpets: predator behavior and defensive features of the shell morphology of the prey. The Biological Bulletin, 171:577-596.

Marsh, C. P. (1986). Impact of avian predators on high intertidal limpet populations. Journal of Experimental Marine Biology and Ecology, 104:185-201.

Menge, B. A. (1975). Brood or broadcast? The adaptive significance of different reproductive strategies in the two intertidal sea stars Leptasterias hexactis and Pisaster ochraceus. Marine Biology, 100:87-100.

Menge, B. A., Berlow, E. L., Blanchette, C. A., Navarrete, S. A., and Yamada, S. B. (1994). The keystone species concept: variation in interaction strength in a rocky intertidal habitat. Ecological Monographs, 64:249-286.

Mercurio, K. S., Palmer, A. R., and Lowell, R. B. (1985). Predator-mediated microhabitat partitioning by two species of visually cryptic, intertidal limpets. Ecology, 66:1417-1425.

Navarrete, S. A., Menge, B. A., and Daley, B. A. (2000). Species interactions in intertidal food webs: prey or predation regulation of intermediate predators? Ecology, 81:2264-2277.

Palmer, A. R. (1990). Predator size, prey size, and the scaling of vulnerability: hatchling gastropods vs. barnacles. Ecology, 71:759-775.

Phillips, D. W. and Castori, P. (1982). Defensive responses to predatory seastars by two specialist limpets, Notoacmea insessa (Hinds) and Collisella instabilis (Gould), associated with marine algae. Journal of Experimental Marine Biology and Ecology, 59:23-30.

Wieters, E. A., Gaines, S. D., Navarrete, S. A., Blanchette, C. A., and Menge, B. A. (2008). Scales of dispersal and the biogeography of marine predator-prey interactions. The American Naturalist, 171:405-417. 